\title{
Genetic Activation of ERK5 MAP Kinase Enhances Adult Neurogenesis and Extends Hippocampus-Dependent Long-Term Memory
}

\author{
Wenbin Wang, ${ }^{1}$ Yung-Wei Pan, ${ }^{2}$ Junhui Zou, ${ }^{1}$ Tan Li, ${ }^{1,3}$ Glen M. Abel, ${ }^{1}$ Richard D. Palmiter, ${ }^{4}$ Daniel R. Storm, ${ }^{5}$ \\ and Zhengui Xia ${ }^{1,2}$ \\ ${ }^{1}$ Toxicology Program in the Department of Environmental and Occupational Health Sciences, University of Washington, Seattle, Washington 98195; \\ ${ }^{2}$ Graduate Program in Molecular and Cellular Biology, University of Washington, Seattle, Washington 98195; ${ }^{3}$ Department of Biochemistry and Genetics, \\ Zhejiang University, Hangzhou, Zhejiang 310058, China; and ${ }^{4}$ Howard Hughes Medical Institute, Department of Biochemistry and ${ }^{5}$ Department of \\ Pharmacology, University of Washington, Seattle, Washington 98195
}

Recent studies have shown that inhibition of adult neurogenesis impairs the formation of hippocampus-dependent memory. However, it is not known whether increasing adult neurogenesis affects the persistence of hippocampus-dependent long-term memory. Furthermore, signaling mechanisms that regulate adult neurogenesis are not fully defined. We recently reported that the conditional and targeted knock-out of ERK5 MAP kinase in adult neurogenic regions of the mouse brain attenuates adult neurogenesis in the hippocampus and disrupts several forms of hippocampus-dependent memory. Here, we developed a gain-of-function knock-in mouse model to specifically activate endogenous ERK5 in the neurogenic regions of the adult brain. We report that the selective and targeted activation of ERK5 increases adult neurogenesis in the dentate gyrus by enhancing cell survival, neuronal differentiation, and dendritic complexity. Conditional ERK5 activation also improves the performance of challenging forms of spatial learning and memory and extends hippocampus-dependent long-term memory. We conclude that enhancing signal transduction of a single signaling pathway within adult neural stem/progenitor cells is sufficient to increase adult neurogenesis and improve the persistence of hippocampus-dependent memory. Furthermore, activation of ERK5 may provide a novel therapeutic target to improve long-term memory.

Key words: adult neurogenesis; ERK5; MAP kinase; memory persistence; spatial learning

\section{Introduction}

Adult neurogenesis in the subgranular zone (SGZ) of the dentate gyrus (DG) is a dynamic process by which new functional neurons are generated from adult neural stem cells/precursors. These neurons integrate into the hippocampal circuitry and contribute to some hippocampus-dependent behaviors (Deng et al., 2010; Ming and Song, 2011). For example, ablation of adult neurogenesis by focal irradiation, antimitotic drugs, or genetic manipulation leads to deficits in the formation of some hippocampusdependent memories (Shors et al., 2001; Imayoshi et al., 2008; Clelland et al., 2009; Deng et al., 2009). Conversely, increased

\footnotetext{
Received Aug. 4, 2013; revised Nov. 26, 2013; accepted Dec. 29, 2013.

Author contributions: W.W., D.R.S., and Z.X. designed research; W.W., Y.-W.P., J.Z., T.L., G.M.A., and R.D.P. performed research; R.D.P. contributed unpublished reagents/analytic tools; W.W., Y.-W.P., J.Z., D.R.S., and Z.X. analyzed data; W.W., D.R.S., and Z.X. wrote the paper.

This work was supported by the National Institutes of Health (Grants R01 MH95840 to Z. X. and R01 NS20498 to D.R.S.) and facilitated by the National Institute of Child Health and Human Development (Grant P3O HD02274). We thank Dr. Chay T. Kuo of the Duke University Medical Center for providing the Nestin-CreER ${ }^{\mathrm{TM}}$ mice, Drs. Toby Cole and Thomas Burbacher of the University of Washington for providing some of the behavior assay equipment and facility, and members of the Xia laboratory for critical reading of the manuscript.

Correspondence should be addressed to Zhengui Xia, PhD, Toxicology Program in the Department of Environmental and Occupational Health Sciences, Box 357234, University of Washington, Seattle, WA 98195. E-mail: zxia@u.washington.edu.

DOI:10.1523/JNEUROSCI.3324-13.2014

Copyright $\odot 2014$ the authors $\quad 0270-6474 / 14 / 342130-18 \$ 15.00 / 0$
}

adult neurogenesis by exercise (Creer et al., 2010), treatment with small molecules (Petrik et al., 2012; Wang et al., 2012), or deletion of the proapoptotic gene Bax (Sahay et al., 2011) improves hippocampus-dependent spatial learning. However, it is unknown whether enhancing adult neurogenesis increases memory persistence. The discovery of signaling pathways that enhance adult neurogenesis may lead to therapeutic strategies for improving memory loss due to aging or injury. Therefore, it is important to develop genetic models that can be used to selectively enhance adult neurogenesis through activation of specific signaling pathways.

In the brain, the ERK5 MAP kinase is specifically expressed in the adult neurogenic regions (Pan et al., 2012b; Pan et al., 2012c; Li et al., 2013). ERK5 is homologous to ERK1/2 but is specifically phosphorylated and activated by MEK5 (English et al., 1995; Zhou et al., 1995). MEK5 is highly specific for ERK5 and does not activate other MAPKs even when overexpressed (English et al., 1995; Zhou et al., 1995). All effects of MEK5 have been attributed to its ability to activate ERK5 (Hayashi and Lee, 2004). The MEK5-ERK5 signaling pathway is activated by neurotrophins in developing neurons (Watson et al., 2001; Liu et al., 2003; Cavanaugh, 2004; Liu et al., 2006; Cundiff et al., 2009; Pan et al., 2012d; Zou et al., 2012), and by neurotrophins and prolactin in adult neural stem/progenitor cells (Pan et al., 2012d; Zou et al., 
A

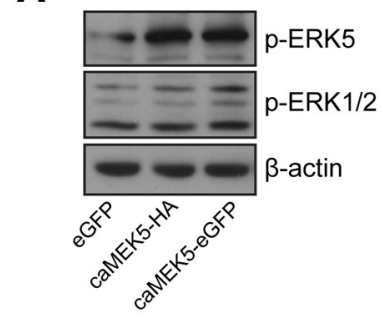

C
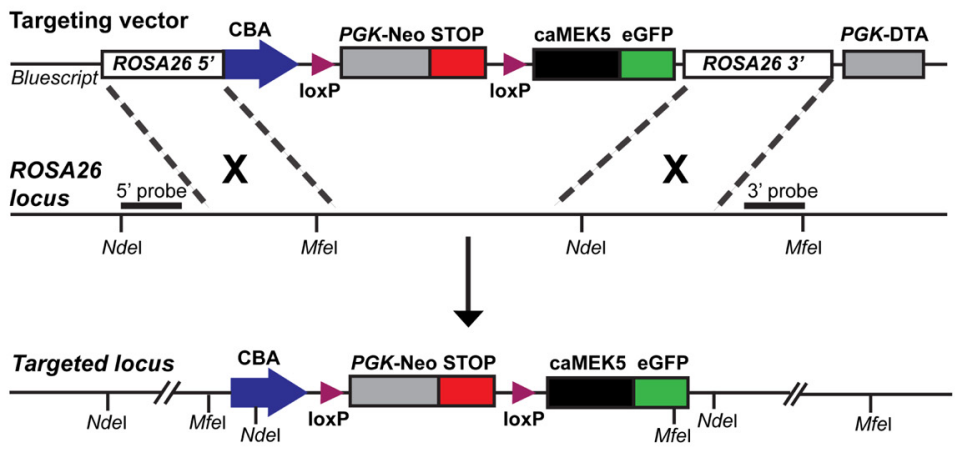

D

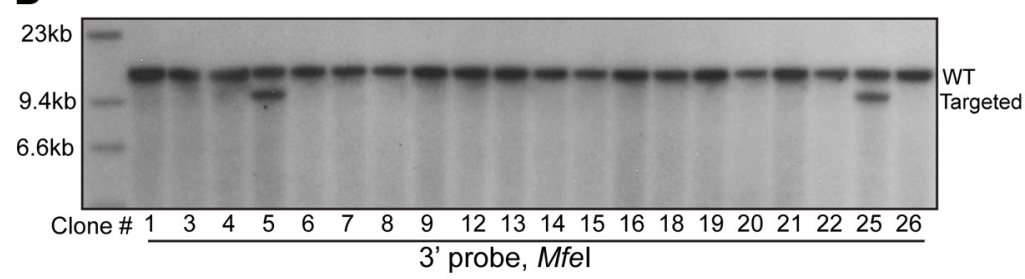

E

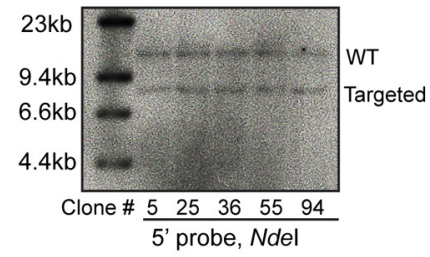

$\mathbf{F}$

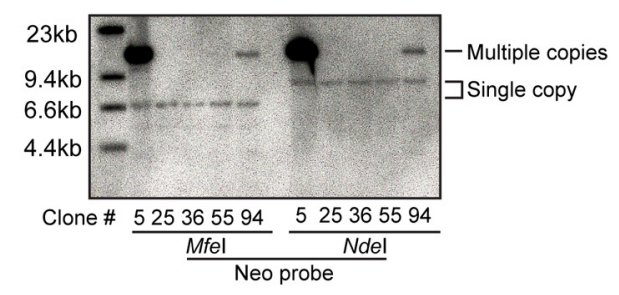

Figure 1. Strategy for generating (ROSA26) CaMEK5-eGFP loxP knock-in mouse line. $A$, Biochemical analysis to confirm that the caMEK5-eGFP fusion protein is constitutively active and functional to activate ERK5. After transfection into NIH3T3 cells, caMEK5eGFP fusion protein stimulates phosphorylation of endogenous ERK5, but not extracellular signal-regulated kinases $1 / 2$ (ERK1/2). Empty vector (eGFP) was used as a negative control and caMEK5-HA as a positive control. $\beta$-actin was used as a loading control. $\boldsymbol{B}$, caMEK5-eGFP fusion protein stimulates the transcriptional activity of MEF2C, a transcription factor and well characterized substrate of ERK5. NIH3T3 cells were transfected with a Gal4-luciferase reporter, an expression vector for Gal4-MEF2C fusion protein, and an expression vector for wild-type ERK5 to measure ERK5 stimulation of MEF2C-mediated transcriptional activity, as described previously (Cavanaugh et al., 2001). The activities of cells cotransfected with eGFP or wild-type (wt) MEK5 were used as baseline controls, whereas the activities of cells transfected with caMEK5-HA were used as a positive control. ${ }^{* * *} p<0.001$ compared with eGFP- or wt-MEK5-transfected cells. C, Schematic diagram depicting the caMEK5-eGFP targeting vector that targets the ROSA26 genomic locus after homologous recombination in mouse ES cells. CDNA sequence encoding caMEK5-eGFP fusion protein was cloned into the targeting vector behind a PGK-Neo-STOP cassette, which was flanked by two loxP sites. The targeting vector contains a CBA promoter, diphtheria toxin (PGK-DTA) for negative selection, and genomic ROSA26 sequences for homologous recombination. D, Southern blot analysis of DNA from 20 ES cell clones digested by Mfel and hybridized with a 3' probe identified 2 targeted ES clones; clone \#5 and clone \#25. Of the 73 total ES cell clones screened, five were positive, targeted clones (data not shown). $\boldsymbol{E}$, Southern analysis of DNA digested by Ndel and hybridized with a 5 ' probe confirmed the five targeted clones. $\boldsymbol{F}$, Southern blot analysis of DNA digested by Mfel or Ndel and hybridized with the Neo probe showed that three of the five ES cell clones were integrated with a single copy of the target gene: clones \#25, \#36, and \#55. The ROSA caMEK5-EGFP mice were derived from clone \#55.

2012; Li et al., 2013; Wang et al., 2013), promoting the survival and neuronal differentiation of newborn neurons both during development and in adult neurogenesis. Inducible and conditional gene targeting of the Mapk7 gene, which encodes ERK5 in adult neurogenesis in the hippocampus and disrupts several forms of hippocampusdependent memory formation (Pan et al., 2012a; Pan et al., 2012c; Pan et al., 2012d). These data indicate that ERK5 may play an important role in the regulation of adult hippocampal neurogenesis.

In this study, we generated a sitespecific knock-in mouse line that allows inducible and conditional expression of a constitutive active MEK5 (caMEK5). This genetic approach provides a unique tool to conditionally activate endogenous ERK5 specifically in the adult neurogenic regions of the brain. We provide evidence that selective activation of ERK5 increases adult neurogenesis in the DG and improves spatial learning and long-term memory persistence.

\section{Materials and Methods}

Generation of the caMEK5-eGFP mice. The caMEK5 knock-in mouse line was generated by targeting the Gt(ROSA)26Sor genomic locus (ROSA26; Soriano, 1999) in embryonic stem (ES) cells derived from a hybrid of 129S6/ SvEvTac $\times$ C57BL/6Ncr (George et al., 2007). ROSA26 is a favored site for integration of transgenes because it can be targeted with high recombination efficiency, provides ubiquitous transgene expression and is not subject to gene-silencing effects(Soriano, 1999). The targeting vector is $\sim 20 \mathrm{~kb}$ long and contains a chicken $\beta$-actin (CBA) promoter followed by a loxP site, a Pgk-Neo expression cassette, a transcriptional stop sequence, another loxP site in the same orientation as the first loxP, and a cDNA sequence encoding caMEK5 fused in frame at the $3^{\prime}$ end with enhanced green fluorescent protein (eGFP) cDNA (caMEK5-eGFP). The $5^{\prime}$ and $3^{\prime}$ arms of the Gt(ROSA)26Sor targeting vector are 7.7 and $4.1 \mathrm{~kb}$, respectively. A diphtheria toxin (DTA) expression cassette was placed after the $3^{\prime}$ arm for negative selection. Mouse G4 ES cells (George et al., 2007) were electroporated with linearized targeting vector using an Amaxa Nucleofector device and grown under positive selection using G418. Cells in which the plasmid was randomly inserted into the chromosome instead of through homologous recombination would express DTA and be killed by the toxin. After drug (G418) selection, 96 of G418-resistant ES cell clones were picked from 96-well culture plates and expanded in 24-well culture plates in the presence of G418 until cells reached confluence. Seventy-three clones grew up and were screened by Southern blot hybridization using a 3' probe on ES cell DNA digested with MfeI. Five positive ES clones were subjected to confirmatory Southern blot analysis using a $5^{\prime}$ probe on NdeIdigested DNA. An internal Neo probe was used on DNA digested with either MfeI or NdeI to detect single-copy integration. Three of the successfully targeted ES clones with single copy integration (clones 
$\# 25$, \#36, and \#55) were then expanded and injected into the blastocoel of 3.5-d-old mouse blastocysts, which were then surgically transplanted to the uterine horns of pseudopregnant females for developing chimeras. Of the 3 ES cell clones injected, only clone \#55 generated chimeras, which were subsequently genotyped by PCR to confirm germline transmission. This line of mice (ROSA caMEK5-eGFP ${ }^{\text {loxP }}$ ) was used for subsequent breeding and analysis.

Animals and tamoxifen administration. The driver Nestin-Cre transgenic mouse line B6.Cg(SJL)-TgN(NesCre) (Jackson Laboratories; Tronche et al., 1999), the driver Nestin$\mathrm{CreER}^{\mathrm{TM}}$ transgenic mouse line (Kuo et al., 2006), and the reporter line Rosa26-loxP-stoploxP-TdTomato (line Ai14; Madisen et al., 2010) have been described previously. The ROSA caMEK5-eGFP ${ }^{\text {loxP }}$ mice were crossed with these driver or reporter mice to generate three knock-in lines: (1) Nestin-Cre:ROSA caMEK5-eGFP ${ }^{\text {loxP }}$ mice, in which caMEK5eGFP is expressed in Nestin-expressing neural stem cells during both the embryonic and adulthood stages; (2) Nestin-CreER ${ }^{\mathrm{TM}}$ :ROSA caMEK5-eGFP ${ }^{\text {loxP/loxP }}$ mice, in which caMEK5eGFP is expressed in Nestin-expressing cells upon tamoxifen administration; and (3) Nestin$\mathrm{CreER}^{\mathrm{TM}}$ :ROSA caMEK5-eGFP ${ }^{\text {loxP }} /$ TdTomato ${ }^{\text {loxP }}$ mice, in which both caMEK5-eGFP and TdTomato, each from one ROSA locus, are expressed in Nestin-expressing cells upon tamoxifen administration.

To induce Nestin-CreER ${ }^{\mathrm{TM}}$-mediated recombination of caMEK5-eGFP and/or TdTomato in neural stem cells in the adult brain, mice ( $8-10$ weeks old) were given freshly made tamoxifen $(200 \mathrm{mg} / \mathrm{kg}$; Sigma) by oral gavage once a day for $7 \mathrm{~d}$ for cellular studies or once a day for $4 \mathrm{~d}$ in each cycle for 3 cycles with 2 -week intercycle intervals for behavior studies, as described previously (Pan et al., 2012a; Pan et al., 2012c). Animals were housed under standard conditions (12 h light/dark cycle) with food and water provided ad libitum. All experimental procedures were approved by the University of Washington Institutional Animal Care and Use Committee.

Bromodeoxyuridine labeling. Bromodeoxyuridine (BrdU; Sigma) was dissolved in sterile saline with $0.007 \% \mathrm{NaOH}$ and administered at $100 \mathrm{mg} / \mathrm{kg}$ by intraperitoneal injection 5 times (every $2 \mathrm{~h}$ ) in $1 \mathrm{~d}$ or once a day for 6 consecutive days, as specified in the figure legends. Mice were killed either $2 \mathrm{~h}$ or 6 weeks after the last BrdU injection to identify BrdU-labeled proliferative cells or BrdU-retaining, adult-born cells.

Immunohistochemistry. The following primary antibodies and dilutions were used for immunohistochemistry or immunocytochemistry: rat monoclonal anti-BrdU (1:500; AbD Serotec); mouse monoclonal anti-NeuN (1:500; Millipore); mouse monoclonal anti- $\beta$-III tubulin (1: 1000; Promega), mouse monoclonal anti-GFAP (1:1000; Millipore); rabbit polyclonal anti-GFP (1:500; Invitrogen); rabbit polyclonal antiDsRed (1:1000; Clontech); goat polyclonal anti-doublecortin (DCX, 1:200; Santa Cruz Biotechnology). Rabbit polyclonal anti-ERK5 (1:500) was generated previously and affinity purified using recombinant MBPERK5 protein (Cavanaugh et al., 2001). Fluorescent secondary antibodies were all used at a dilution of 1:500 (Invitrogen).

B the closely related ERK1/2.
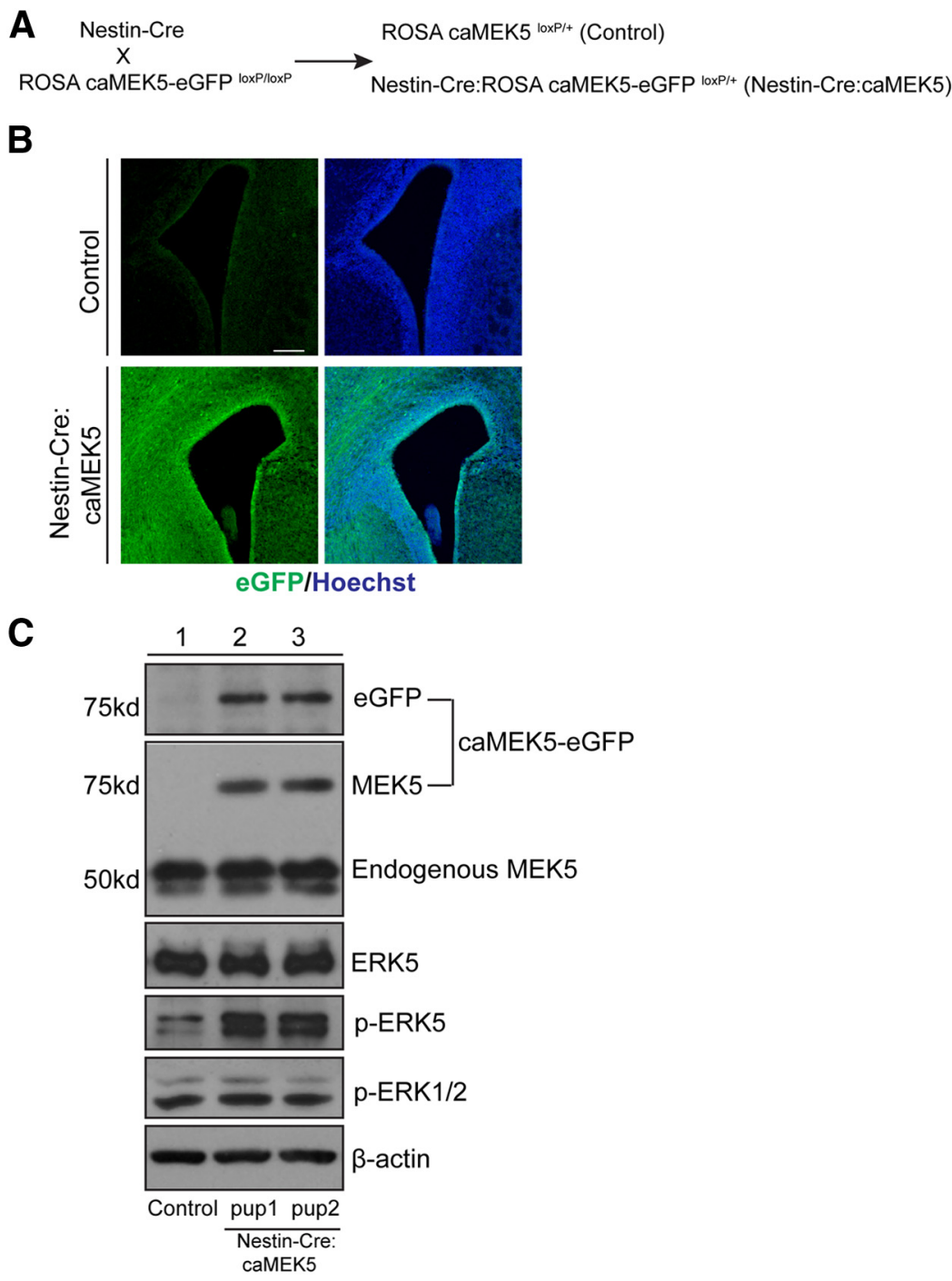

igure 2. caMEK5-eGFP specifically activates endogenous ERK5, but not the closely related ERK1/2, in the brain after Cremediated recombination. $\boldsymbol{A}$, Breeding scheme for generating Nestin-Cre:caMEK5 mice to express caMEK5-eGFP in the brain (ROSA26)caMEK5-eGFP loxP/loxP mice were crossed with the Nestin-Cre mice to generate Nestin-Cre:caMEK5-eGFP loxP/+ mice, designated Nestin-Cre:caMEK5 mice. caMEK5-eGFP loxP/+ littermates were controls. $\boldsymbol{B}$, Fluorescent images showing caMEK5-eGFP expression around the lateral ventricle regions of the brain of newborn Nestin-Cre:caMEK5 pups, but not that of the controls. Green, eGFP; blue, Hoechst. Scale bars, $100 \mu \mathrm{m}$ in all images. C, Western blot analysis of newborn mouse brain lysates demonstrates expression of caMEK5-eGFP fusion protein in brain homogenates of Nestin-Cre:caMEK5 (pups \#1 and \#2), but not that of control littermate pups. The anti-MEK5 antibody recognizes both endogenous MEK5 and caMEK5-eGFP fusion proteins. Phosphorylated ERK5 ( $p$-ERK5) and ERK1/2 (p-ERK1/2) were detected by an antibody that recognizes both on the same blot. Total ERK5 and $\beta$-actin were used as loading controls. Expression of caMEK5-eGFP fusion protein induced phosphorylation of endogenous ERK5, but not

Mice were killed and perfused transcardially with $4 \%$ paraformaldehyde (PFA) in phosphate-buffered saline (PBS, pH 7.4). Brains were postfixed in $4 \%$ PFA overnight, followed by $30 \%(\mathrm{w} / \mathrm{v})$ sucrose/PBS solution at $4^{\circ} \mathrm{C}$ until brains sank. Immunohistochemistry for all except eGFP was performed on $30-\mu \mathrm{m}$-thick coronal brain sections using a free-floating antibody staining method as described previously (Wang et al., 2013). For eGFP or phospho-ERK1/2 staining, a tyramide signal amplification (TSA; PerkinElmer) kit was used (Lagace et al., 2007). Briefly, tissues were rinsed with TBS $(0.1 \mathrm{~m}$ Tris- $\mathrm{HCl}, \mathrm{pH} 7.4,0.9 \% \mathrm{NaCl})$ for $3 \times$ $10 \mathrm{~min}$, followed by quenching endogenous peroxidase with $1 \% \mathrm{H}_{2} \mathrm{O}_{2} /$ TBS $2 \times 15 \mathrm{~min}$. Tissues were blocked in $10 \%$ goat serum, $1 \%$ BSA in TBST (TBS with $0.1 \%$ Tween 20 ) for $1-2 \mathrm{~h}$ at room temperature, followed by incubation with primary antibodies (rabbit anti-GFP; Invitrogen; rabbit anti-phospho-ERK1/2 [Thr202/Tyr204]; Cell Signaling Technology) diluted at 1:2000 or 1:4000 in blocking buffer overnight at 
A

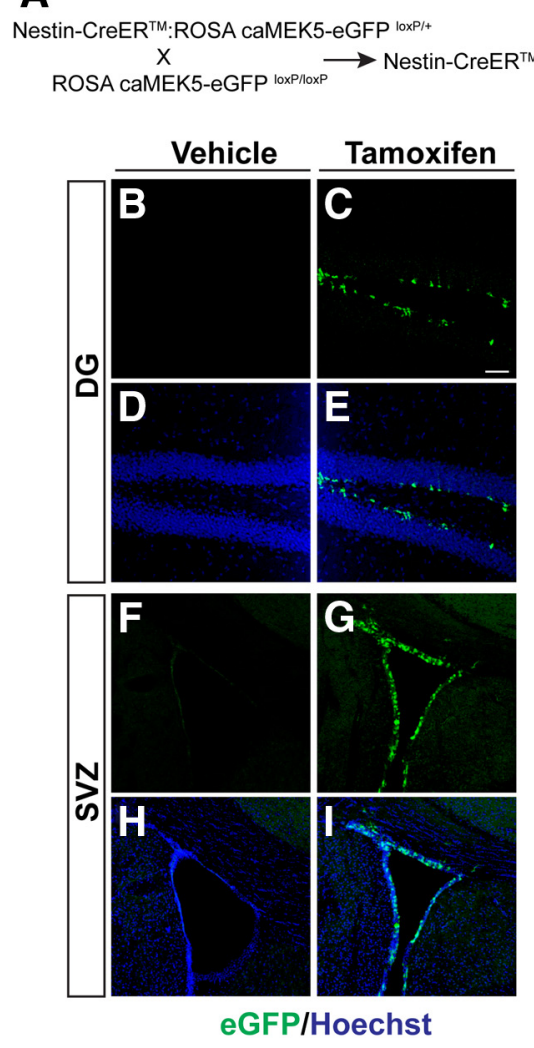

Figure 3. caMEK5-eGFP expression is conditionally induced in the adult neurogenic regions of the mouse brain. $A$, Experimental scheme for generating NesCreER:caMEK5 mice. Nestin-CreER ${ }^{T M}$ :CaMEK5-eGFP loxP/loxP mice were generated by crossing (ROSA26) caMEK5-eGFP loxP/loxP with Nestin-CreER ${ }^{\text {TM }}$ mice. Eight-week-old mice were treated with vehicle (corn oil) or tamoxifen orally once a day for $7 \mathrm{~d}$ and perfused 3 weeks after the last tamoxifen dosing. $\boldsymbol{B}$ - I, Anti-eGFP immunostaining (green) demonstrates expression of caMEK5-eGFP in the SGZ of the DG and the SVZ of NesCreER:caMEK5, but not control mice. Scale bars, $50 \mu \mathrm{m}$ in all panels.

$4^{\circ} \mathrm{C}$. After washing $4 \times 10 \mathrm{~min}$ with TBST, tissues were incubated with secondary antibody (biotinylated goat anti-rabbit; Vector Laboratories) at a 1:250 dilution for $2 \mathrm{~h}$ at room temperature. Tissues were further washed for $4 \times 10 \mathrm{~min}$, followed by incubation with streptavidin-HRP (TSA kit; PerkinElmer) at 1:200 or Extra-Avidin (Sigma) at 1:400 for 30 $\mathrm{min}$ at room temperature. Staining was visualized with Cy3-Tyramide or fluorescein-tyramide (NEL704A or NEL701; PerkinElmer) 1:75 for $8 \mathrm{~min}$ in the dark at room temperature.

Confocal imaging and analysis. All images were captured with an Olympus Fluoview-1000 laser scanning confocal microscope with numerical aperture (NA) 0.75 with $10 \times$ or $20 \times$ lenses or an NA $1.3,40 \times$ oil-immersion lens. Optical $Z$-sections $(1 \mu \mathrm{m})$ were collected and processed using ImageJ software (NIH). Images were uniformly adjusted for color, brightness, and contrast with Adobe Photoshop CS6.

Analysis of dendritic morphology. All images were captured using Olympus Fluoview-1000 laser scanning confocal microscope with NA $1.35,60 \times$ oil-immersion lens or NA 4.0, $100 \times$ oil-immersion lens. Optical $Z$-series $(0.5 \mu \mathrm{m})$ were collected and processed using Fiji software (NIH). Quantification of dendritic length and number of dendrites were performed as described previously (Li et al., 2013), using Simple Neurite Tracer (NIH), with at least 16 individual neurons analyzed per genotype. Sholl analysis to determine number of crossings was measured using the Sholl Analysis function in Fiji (NIH). Skeleton images of individual neurons were traced using Simple Neurite Tracer in 3D across a $Z$-series and projected as a maximum projection $2 \mathrm{D}$ image. For quantification of dendritic spines, at least 40 dendrites per genotype were measured for length using Simple Neurite Tracer and the total number of spines per dendrite was quantified manually after capture at a $300 \times$ digital zoom. For all quantifications, the experimenter was blinded to the genotype.
Quantification of immunostained cells. Every eighth serial coronal section $(30 \mu \mathrm{m})$ was immunostained and immunoreactive-positive cells in the granule cell layer of the DG along the septotemporal axis were counted with the experimenter blinded to mice genotypes or treatments, as described previously (Pan et al., 2012c). Resulting numbers were multiplied by 8 to obtain an estimated total number of labeled cells per dentate gyrus. For colocalization analysis, cells were analyzed per marker combination using confocal microscopy. At least 200 immunopositive cells from each mouse were selected randomly and the presence of overlapping fluorescent signals in a $Z$-series stack of a single cell was considered as an indication of a double-positive cell.

Western analysis. Whole-brain lysates were extracted from newborn pups. Briefly, brains were dissected and cut into halves along the midline. A single half brain of each pup was mechanically homogenized with $150 \mu$ l of Triton-X 100 lysis buffer on ice using a homogenizer until tissue became homogenized without any remaining clumps. Tissue fragments were then transferred into a $1.5 \mathrm{ml}$ tube and added with an additional $300 \mu$ lof lysis buffer. Samples were kept on ice for 15 min with frequent vortexing, followed by centrifugation for $10 \mathrm{~min}$ at $13,000 \times \mathrm{g}$. Supernatant was collected and stored in $-80^{\circ} \mathrm{C}$ until use. Protein concentration was determined by a BCA protein assay (Pierce). Samples containing $10 \mu \mathrm{g}$ of protein were separated on $10 \%$ SDS-PAGE gels and transferred to PVDF membrane (Millipore), followed by immunoblotting and ECL Plus (GE Healthcare) detection. The following antibodies were used as primary antibodies for Western analysis: rabbit anti-ERK5 (1:20,000; Cavanaugh et al., 2001), rabbit anti-phosphoERK5 (1:1000, Thr218/Tyr220; Cell Signaling Technology), rabbit anti-GFP (1:1000; Invitrogen), rabbit anti-MEK5 (1:1000; Enzo Life Sciences), and mouse anti- $\beta$-actin (1:20,000; Sigma).

Open field test. The open field test, which measures the baseline locomotor activity and anxiety, was performed as described previously (Pan et al., 2012c). TruScan Photo Beam Tracking arena (Coulbourn) was used for the test and TruScan 2.02 software (Coulbourn) was used for data analysis. Mice were tested on two consecutive days in the same arena.

Novel object recognition and novel location recognition. This assay was done as described previously with slight modifications (Pan et al., 2012c). A 5 min session was used throughout the experiment for both training and tests. The same cohort of mice was used for testing 1,24, and $48 \mathrm{~h}$ memory using different sets of objects. A separate cohort of mice was used for the $72 \mathrm{~h}$ test. Exploratory activity for each toy was recorded for both training and testing sessions and analyzed by experimenters blinded to genotypes.

Novel location recognition was performed as described previously (Okamura et al., 2011). Briefly, two identical objects were placed in distinct corners of the open field, where a mouse was allowed to explore for 5 min during the training session. To exclude spatial preferences of object location, alternating corners were chosen for object presentation during training. Testing was performed 1, 48, or $72 \mathrm{~h}$ after training, during which time one of the two objects (randomly chosen) was moved to a different corner and the time spent exploring both objects over $5 \mathrm{~min}$ was quantified. Increased time spent exploring the object in the novel location was interpreted as successful retention of spatial memory for the object that had not been moved. Different sets of objects were used for each test of the same cohort of mice. 

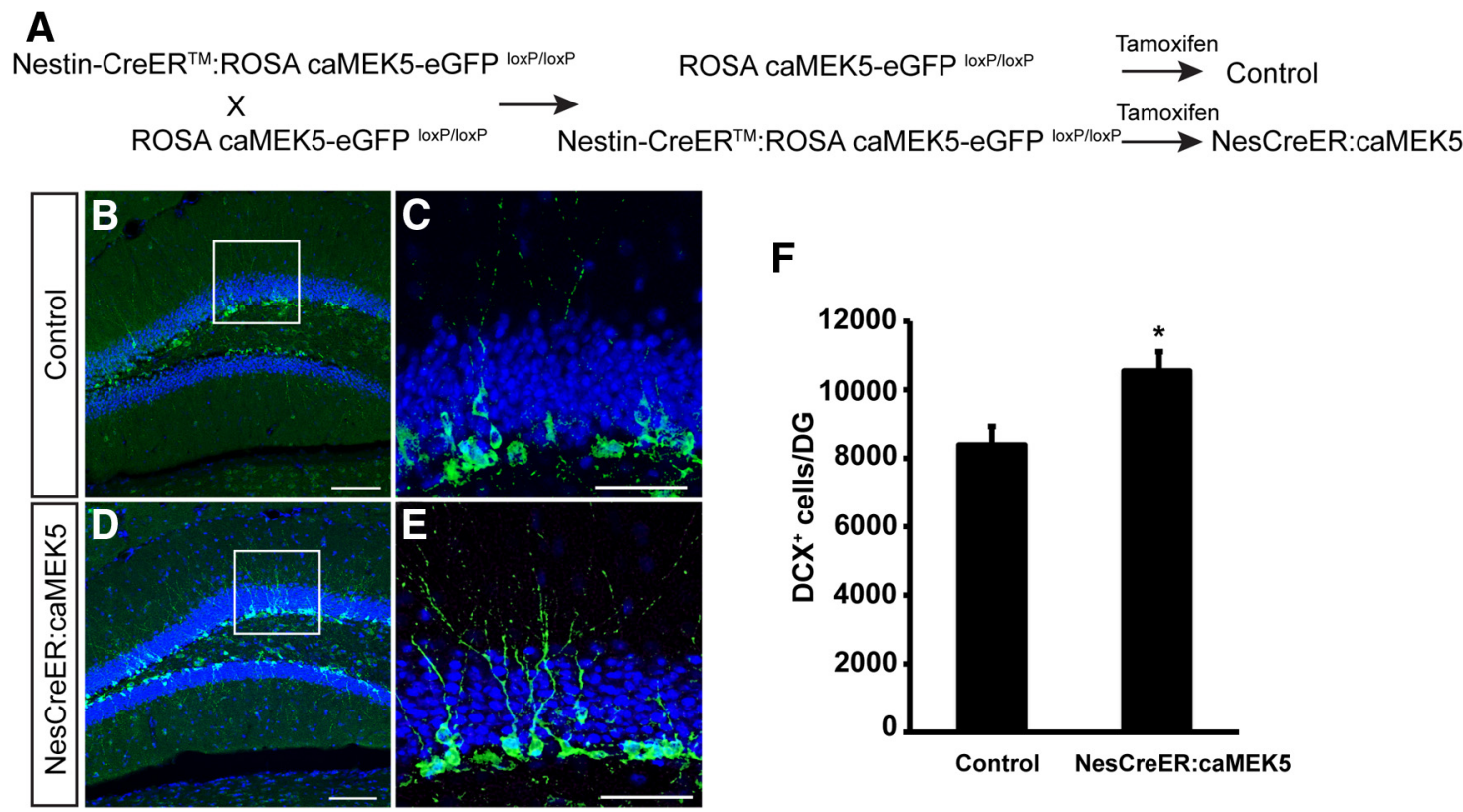

$\mathbf{F}$

DCX/Hoechst

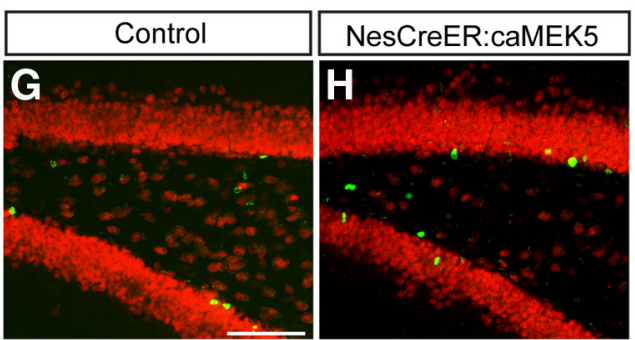

BrdU/NeuN

J 2 h post-BrdU injection

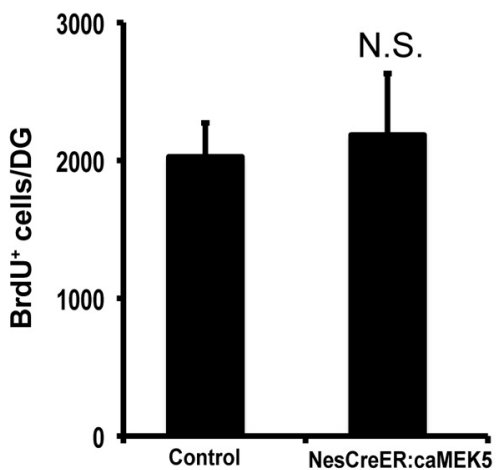

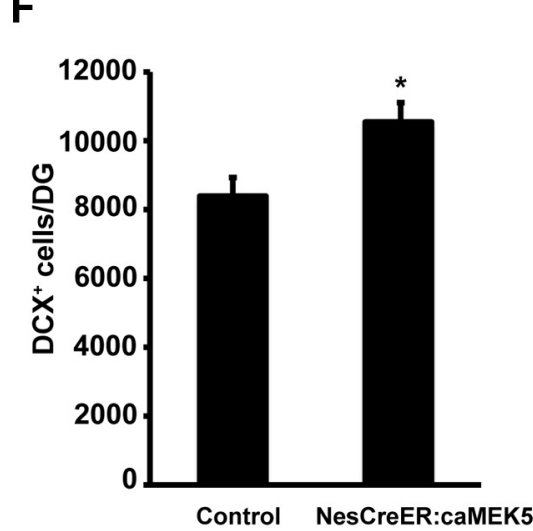

I

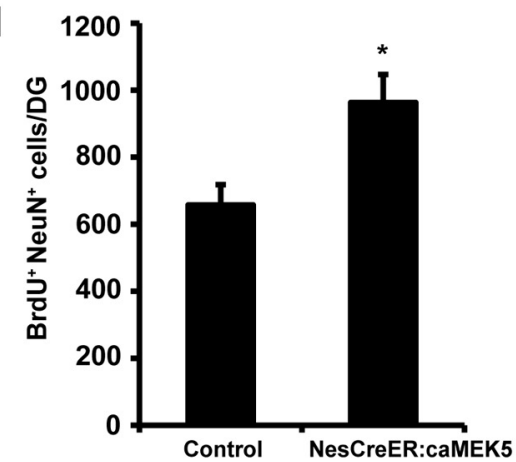

$\mathbf{K}$ 6-week post-BrdU injection

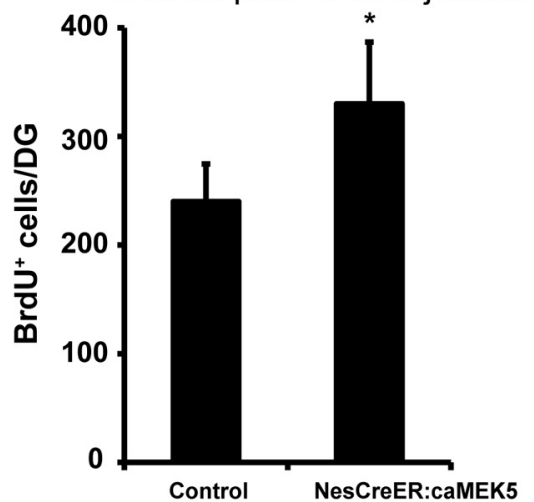

Figure 4. Induced conditional activation of ERK5 in the adult neurogenic regions of NesCreER:caMEK5 mice enhances hippocampal neurogenesis. $\boldsymbol{A}$, Breeding and tamoxifen treatment scheme for generating NesCreER:caMEK5 and control littermates used in this and all behavior experiments. $\boldsymbol{B}$ - $\boldsymbol{E}$, Representative DCX immunostaining images (green) from the SGZ of control ( $\boldsymbol{B}, \boldsymbol{C}$ and NesCreER:caMEK5 $(\boldsymbol{D}, \boldsymbol{E})$ mouse brains, perfused 2 weeks after the last day of a $7 \mathrm{~d}$ tamoxifen treatment. Images in $\boldsymbol{C}$ and $\boldsymbol{E}$ are enlarged areas from boxed regions in $\boldsymbol{B}$ and $\boldsymbol{D}$, respectively. Scale bars, $50 \mu \mathrm{m} . \boldsymbol{F}$, Quantification of DCX ${ }^{+}$cells in each DG. $n=4$ mice/group; ${ }^{*} p<0.05$. $\boldsymbol{G}, \boldsymbol{H}$, Representative BrdU (green) and NeuN (red) costaining from the SGZ of control ( $\boldsymbol{G}$ ) and NesCreER:caMEK5 $(\boldsymbol{H})$ mouse brains perfused 7.5 weeks after the last day of a $7 \mathrm{~d}$ tamoxifen treatment. BrdU was injected once a day for $6 \mathrm{~d}$ starting $4 \mathrm{~d}$ after the last day of tamoxifen. Scale bar, $100 \mu \mathrm{m}$. $\boldsymbol{I}$, Quantification of data from $\mathbf{G}$ and $\boldsymbol{H}$, the number of NeuN ${ }^{+}$and BrdU ${ }^{+}$cells per DG. $n=5$ mice/group; ${ }^{*} p<0.05$. $\boldsymbol{J}$, Ten days after the last tamoxifen injection, BrdU was injected every $2 \mathrm{~h} \times 5$ and mice were perfused $2 \mathrm{~h}$ after the last injection. Total BrdU ${ }^{+}$cells in each DG of mice was quantified. $n=3-4$ mice/group. $\boldsymbol{K}$, Ten days after the last tamoxifen injection, BrdU was injected every $2 \mathrm{~h} \times 5$ and mice were perfused 6 weeks after the last BrdU injection. Total BrdU ${ }^{+}$cells in each DG of mice were quantified. $n=5$ mice/group; ${ }^{*} p<0.05$. BrdU was injected every $2 \mathrm{~h} \times 5$ for $\boldsymbol{J}$ and $\boldsymbol{K}$.

The discrimination index is defined as the difference between the exploration time for the novel object or object in the novel location and that for the familiar object or location, respectively, divided by total exploration time.
Morris water maze assay. The classic Morris water maze assay (MWM) assay was done with the same setting (room, pool, platform, extra-maze cues, etc.) as we described previously (Pan et al., 2012c). Two cohorts of mice were tested for this assay. For Cohort 1, mice were trained two trials 

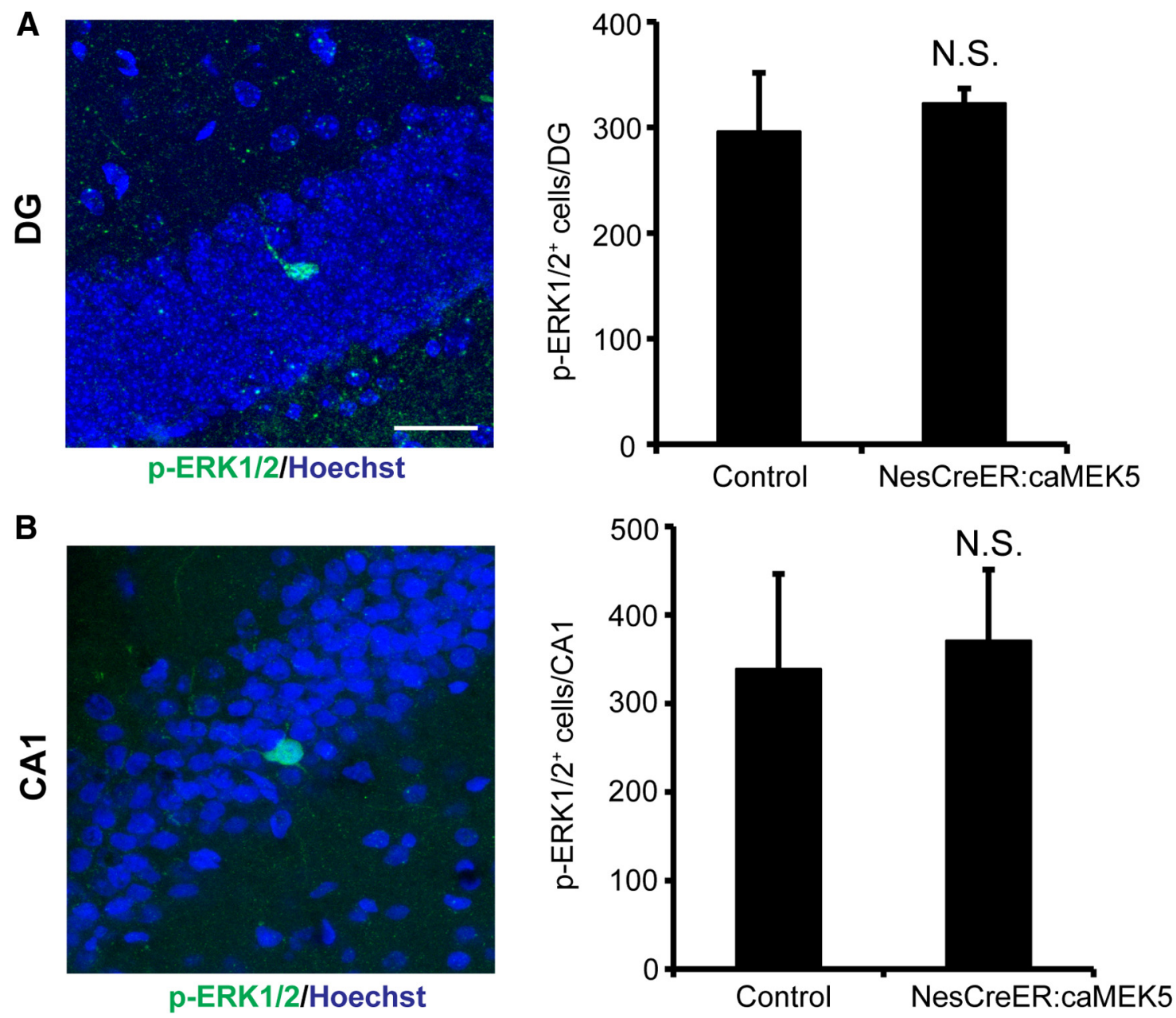

Figure 5. Induced conditional caMEK5 expression in the adult neurogenic regions does not alter ERK1/2 activity. $\boldsymbol{A}$, Representative phospho-ERK1/2 (p-ERK1/2) staining (green) in the DG and quantification of $\mathrm{p}$-ERK1/2 ${ }^{+}$cells per DG. $\boldsymbol{B}$, Representative phospho-ERK1/2 (p-ERK1/2) staining (green) in the pyramidal cell layer of the CA1 region and quantification of p-ERK $1 / 2{ }^{+}$cells per CA1. $n=3$ mice/group. N.S., not significant. Scale bar, $25 \mu \mathrm{m}$.

per day with $40 \mathrm{~s}$ swim and $15 \mathrm{~s}$ consolidation on the escape hidden platform for each trial until the learning curve for both control and knock-in mice plateaued ( $10 \mathrm{~d}$ for this cohort). One day after the last day of training (d11), a probe test was performed as above. On d12 ( $1 \mathrm{~d}$ after the probe test), mice were subjected to reversal training for 2 trials a day and 8 consecutive days where the escape hidden platform was moved from the initial quadrant (Q1) to the opposite quadrant (Q3). A reversal probe test was performed $1 \mathrm{~d}$ after the last day of reversal training. Two days after the reversal probe test, a visible platform test was performed as described previously (Pan et al., 2012c). For Cohort 2, mice were trained 4 trials per day with $40 \mathrm{~s}$ swim followed by $15 \mathrm{~s}$ consolidation on the hidden escape platform for each trial for 8 consecutive days (d1-d8). One day after the last training trial (d9), a probe test was performed in which the hidden platform was removed and mice were allowed to swim for $60 \mathrm{~s}$ in search of the platform. The same probe tests were repeated three more times on $\mathrm{d} 23$, $\mathrm{d} 37$, and $\mathrm{d} 51$ (2, 4, and 6 weeks after the first probe test, respectively).

Statistical analysis. All data were expressed as mean \pm SEM. Student's $t$ test (two-tailed) was used to analyze in vivo cellular data and novel object recognition. A $t$ test or ANOVA with repeated measures was used to analyze data for MWM training tests. One-way ANOVA with post hoc analysis was used for probe tests. In the figures, NS is not significant; ${ }^{*} p<$ $0.05 ;{ }^{* *} p<0.01 ;$ and ${ }^{* * *} p<0.001$.

\section{Results}

Generation of an ERK5-activating knock-in mouse line To generate a mouse line that can be used to activate ERK5 in a temporally and spatially controlled manner, a cDNA se- quence encoding a constitutive active MEK5 (caMEK5; Kato et al., 1997; Liu et al., 2006) was first cloned into the pEGFP-N1 vector to generate the caMEK5-eGFP fusion protein. In vitro biochemical analysis showed that this caMEK5eGFP fusion protein is constitutively active and functional, as evidenced by its selective phosphorylation of ERK5, but not the closely related ERK1/2 (Fig. $1 A$ ), and by its ability to stimulate transcriptional activity of MEF2C, a well characterized ERK5 substrate (Kato et al., 1997; Liu et al., 2003; Fig. 1B).

The caMEK5-eGFP cassette was then cloned into the Gt(ROSA) 26Sor such that it would only be expressed by removal of a floxed Pgk-Neo STOP cassette (Fig. 1C). The ROSA caMEK5-eGFP mice with homologous recombination of this construct into the Gt(ROSA)26Sor genomic locus (ROSA26) were generated as described in the Materials and Methods (Fig. 1D-F).

caMEK5-eGFP gene specifically activates ERK5 in the brain To ascertain whether the caMEK5-eGFP fusion protein can activate endogenous ERK5 in the brain of knock-in mice, we crossed ROSA caMEK5-eGFP ${ }^{\text {loxP/loxP }}$ mice with Nestin-Cre mice (Tronche et al., 1999) to generate Nestin-Cre:caMEK5 mice that express a single copy of caMEK5-eGFP in all Nestin-expressing cells and their progeny during brain development (Fig. 2A). Immunohistochemistry of the brain sections revealed that NestinCre:caMEK5 newborn pups expressed caMEK5-eGFP, whereas 
their control littermates (without NestinCre) did not (Fig. 2B), confirming the conditional expression of caMEK5-eGFP. Western analysis confirmed expression of the caMEK5-eGFP fusion protein in the brains of Nestin-Cre:caMEK5 pups but not of control littermates (Fig. 2C). The expression level of caMEK5-eGFP fusion protein was approximately half that of the endogenous MEK5, suggesting that the transgene is not overexpressed compared with the endogenous MEK5. Significantly, expression of caMEK5-eGFP increased phospho-ERK5, but not the closely related phospho-ERK1/2, demonstrating that the caMEK5-eGFP fusion protein is constitutively active and can specifically activate endogenous ERK5 in knock-in mouse brains.

Inducible and conditional activation of ERK5 in the adult neurogenic regions increases hippocampal neurogenesis by promoting cell survival and neuronal differentiation

To induce expression of caMEK5-eGFP in adult brains, ROSA caMEK5-eGFP mice were bred with Nestin-CreER ${ }^{\mathrm{TM}}$ mice (Kuo et al., 2006). Mice treated with tamoxifen of the resulting line, NestinCreER $^{\text {TM}}$ :ROSA caMEK5-eGFP, are designated NesCreER:caMEK5 (Fig. $3 A$ ). Cre-mediated recombination and expression of caMEK5-eGFP in these mice should occur specifically in Nestinexpressing neural stem cells and their progeny. Indeed, a 7d tamoxifen treatment induced caMEK5-eGFP expression specifically in the two neurogenic regions of adult mice, the DG and the SVZ, as evidenced by eGFP immunofluorescence (Fig. $3 C, E, G, I)$. Vehicle-treated control mice did not show any GFP staining in these regions (Fig. $3 B, D, F, H$ ), demonstrating the tight control of Nestin-CreER ${ }^{\mathrm{TM}}$-mediated recombination in these mice.

The effect of caMEK5 on adult neurogenesis was examined using brain sections from NesCreER:caMEK5 mice and ROSA caMEK5-eGFP ${ }^{\text {loxP/loxP }}$ littermates similarly treated with tamoxifen as controls (Fig. 4A). There was a statistically significant increase in the total number of DCX ${ }^{+}$cells in the DG of NesCreER:caMEK5 mice at 2 weeks after the last tamoxifen administration $(p=0.047$; Fig. $4 B-F)$. In a separate set of experiments, mice were dosed with tamoxifen for $7 \mathrm{~d}$ and perfused 7.5 weeks after the last dose of tamoxifen. To label adultborn cells, BrdU was injected daily for $6 \mathrm{~d}$ starting $4 \mathrm{~d}$ after the last dose of tamoxifen. The DG of NesCreER:caMEK5 mice had an increased number of adult-born, mature neurons $\left(\mathrm{NeuN}^{+}\right.$and $\mathrm{BrdU}^{+}$cells, $p=0.019$; Fig. $\left.4 G-I\right)$. These data suggest that conditional ERK5 activation by caMEK5 enhances adult hippocampal neurogenesis and increases the number of adult-born neurons in the SGZ.

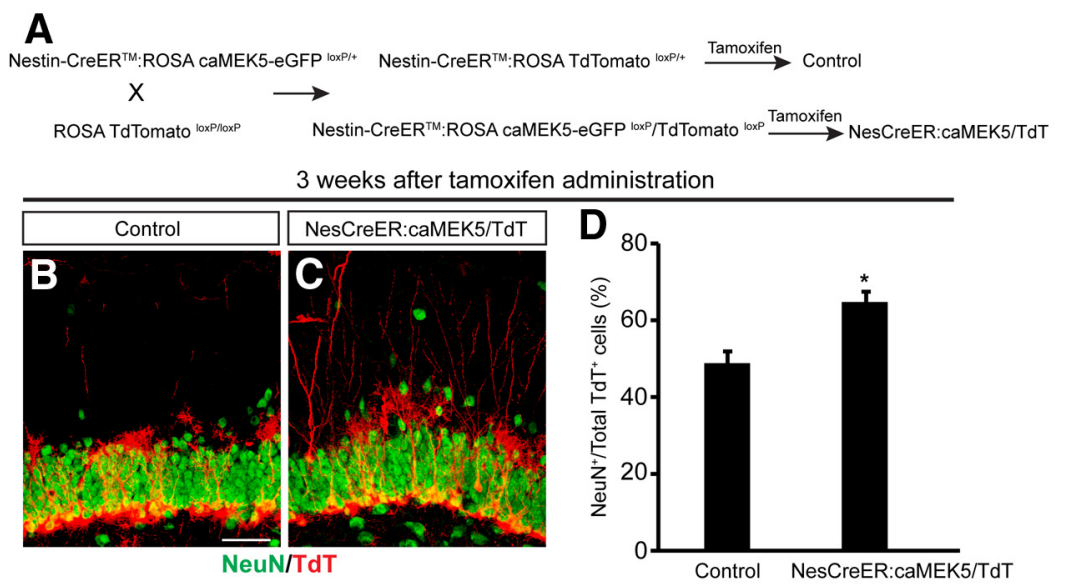

7 weeks after tamoxifen administration
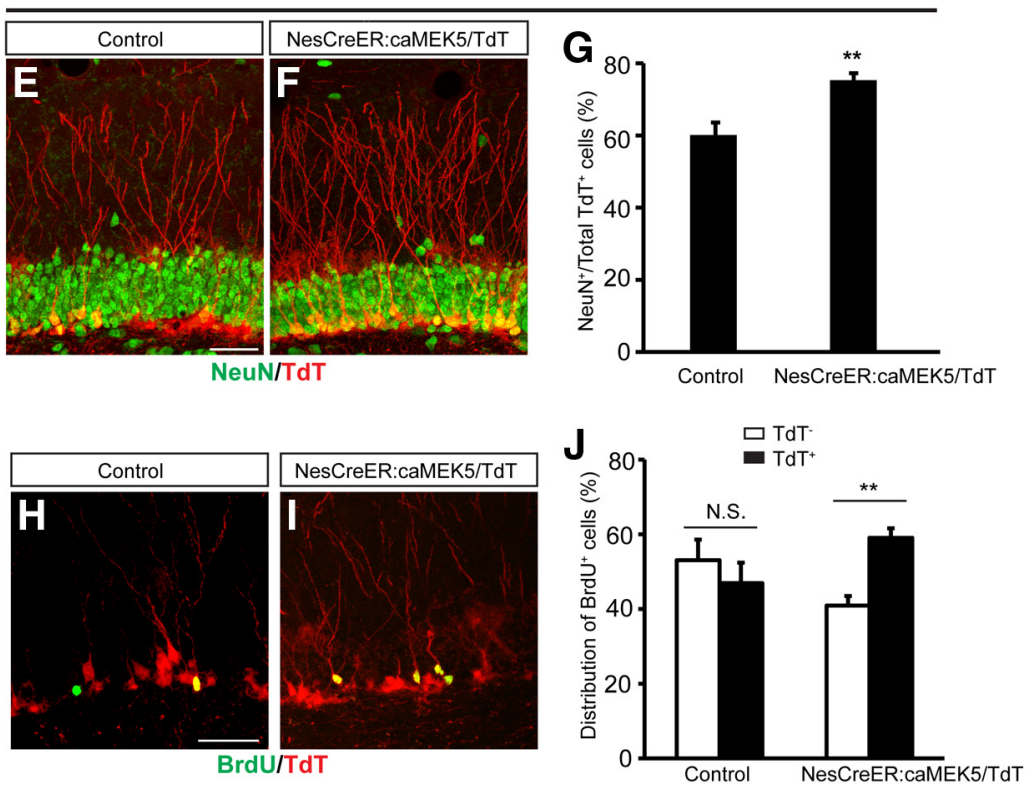

Figure 6. Expression of a single copy of the caMEK5-eGFP gene in the adult neurogenic regions of NesCreER:caMEK5/TdT mice is sufficient to increase hippocampal neurogenesis. $\boldsymbol{A}$, Breeding and tamoxifen treatment scheme for generating NesCreER: caMEK5/TdT and control littermate mice. Mice were treated with tamoxifen daily for $7 \mathrm{~d}$ and perfused either 3 or 7 weeks later. $\boldsymbol{B}$, $\boldsymbol{C}$, Representative NeuN (green) and TdTomato (red) costaining from the SGZ of control (B) and NesCreER:caMEK5/TdT (C) mouse brains perfused 3 weeks after the last dose of tamoxifen. D, Quantification of NeuN ${ }^{+}$and TdTomato $^{+}\left(\mathrm{TdT}^{+}\right.$) cells per DG from data in $\boldsymbol{B}$ and $\boldsymbol{C}$. $\boldsymbol{E}, \boldsymbol{F}$, Representative NeuN (green) and TdTomato (red) costaining from the SGZ of control $(\boldsymbol{E})$ and NesCreER: caMEK5/TdT $(\boldsymbol{F})$ mouse brains perfused 7 weeks after the last dose of tamoxifen. $\mathbf{G}$, Quantification of NeuN ${ }^{+}$and TdTomato ${ }^{+}$ $\left(\mathrm{TdT}^{+}\right.$) cells per DG from data in $\boldsymbol{E}$ and $\boldsymbol{F} . \boldsymbol{H}, \boldsymbol{I}$, Representative BrdU (green) and TdTomato (red) costaining from the SGZ of control $(\boldsymbol{H})$ and NesCreER:caMEK5/TdT $(\boldsymbol{I})$ mouse brains perfused 7 weeks after the last dose of tamoxifen. $\boldsymbol{J}$, Quantification of data in $\boldsymbol{H}$ and $I$ for the relative distribution of $B r d U{ }^{+}$cells among recombined $\mathrm{TdT}^{+}$cells versus BrdU ${ }^{+}$cells among non-recombined $\mathrm{TdT}^{-}$ cells. Scale bars, $100 \mu \mathrm{m} . n=4-5$ mice/group; ${ }^{*} p<0.05$; ${ }^{* *} p<0.01$.

We investigated whether the increased number of adult born neurons is a result of increased proliferation, neuronal differentiation, and/or cellular survival. To label actively proliferating cells, control and NesCreER:caMEK5 mice were injected with BrdU every $2 \mathrm{~h} \times 5$ and perfused $2 \mathrm{~h}$ after the last injection. There was no significant increase of $\mathrm{BrdU}^{+}$cells in the DG of NesCreER:caMEK5 mice compared with control littermates ( $p=0.75$; Fig. $4 J)$, suggesting that increased proliferation is an unlikely mechanism of caMEK5-induced adult neurogenesis.

In a separate set of experiments, control and NesCreER: caMEK 5 mice were injected with BrdU every $2 \mathrm{~h} \times 5$ and perfused 6 weeks later to determine the survival of adult-born cells. 

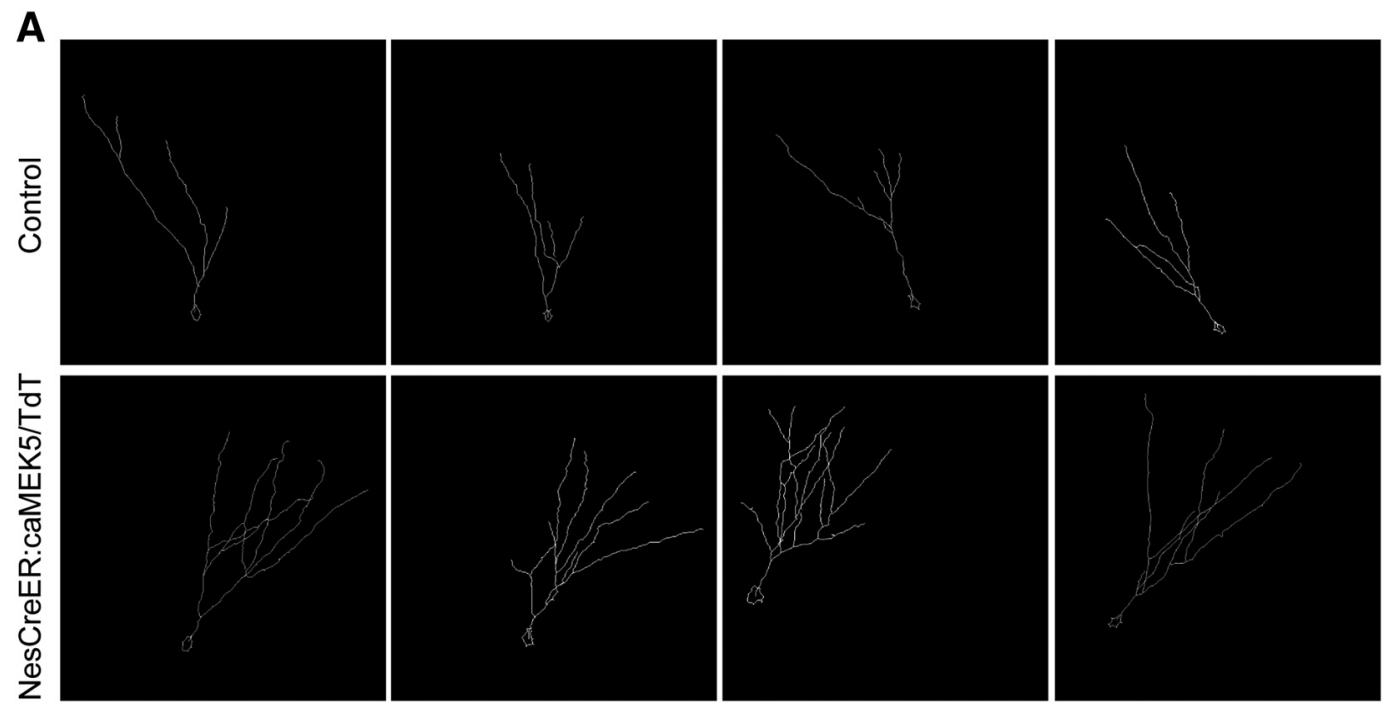

B

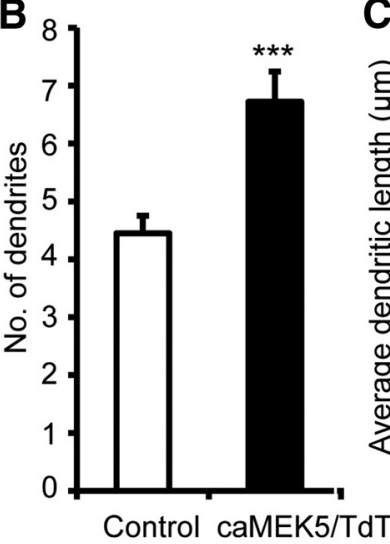

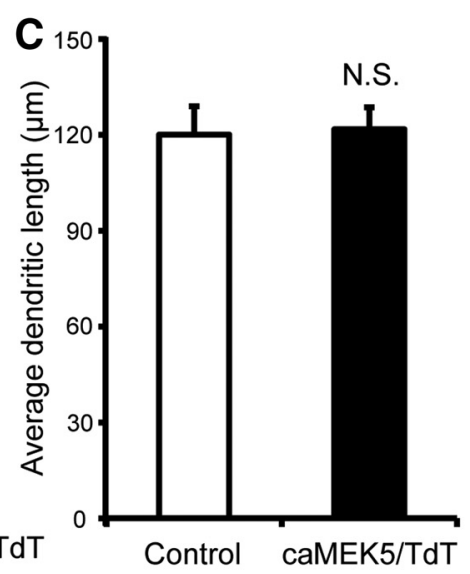

D
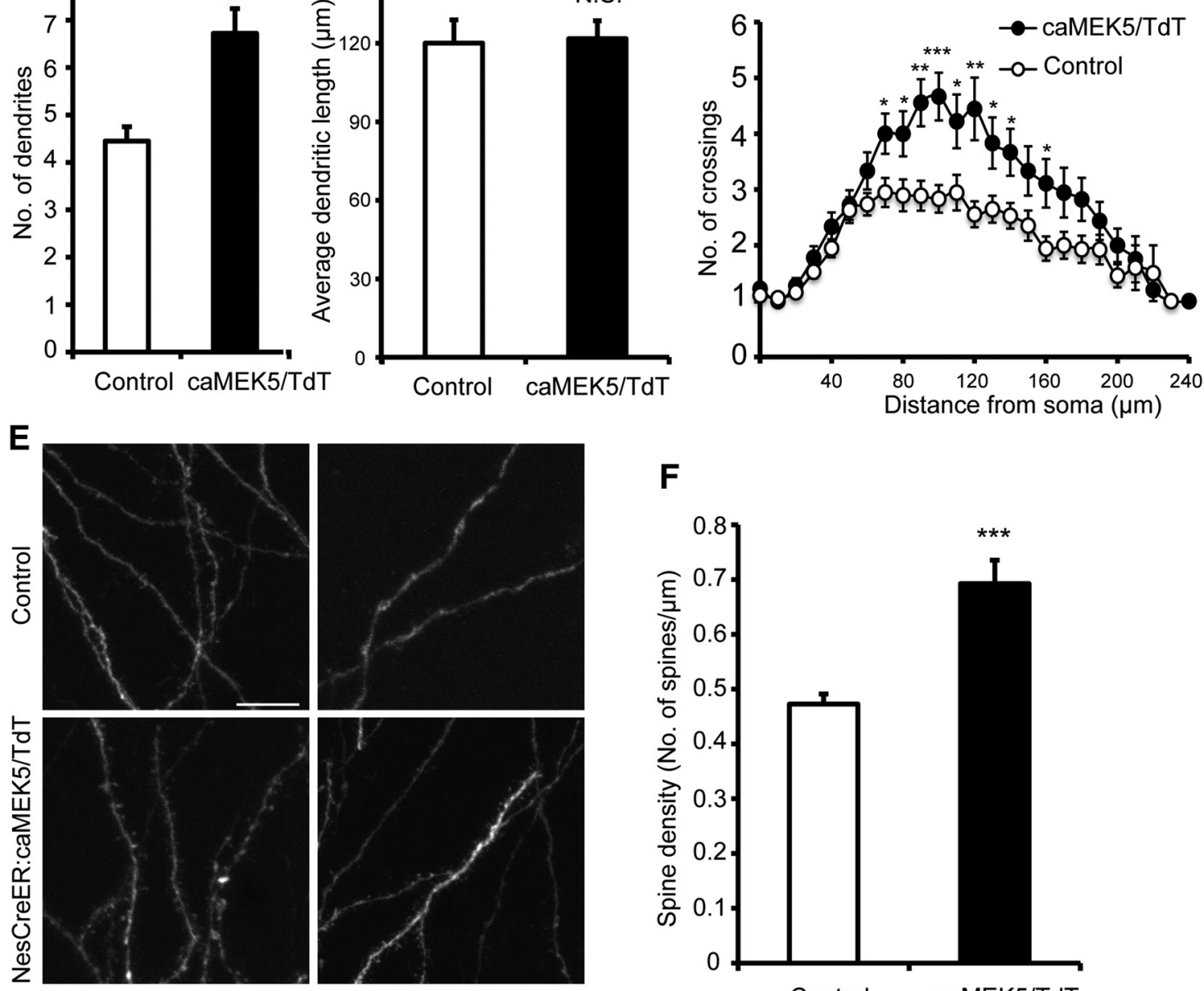

$\mathbf{F}$

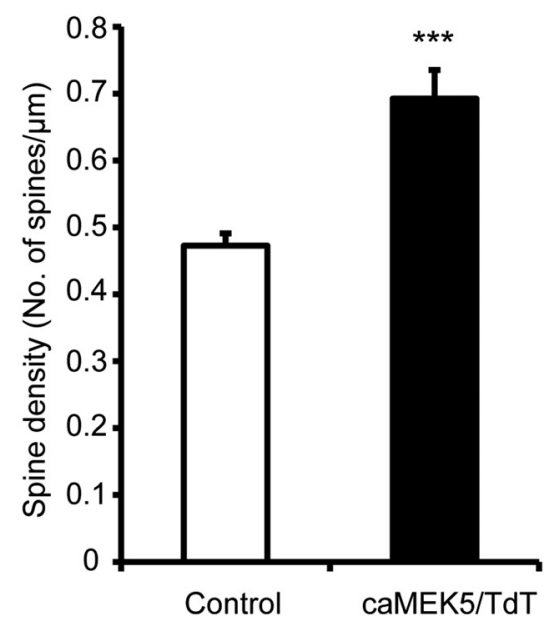

Figure 7. Induced conditional ERK5 activation enhances dendritic complexity of the adult-born neurons in the DG. Mice were treated with tamoxifen daily for $7 \mathrm{~d}$ and perfused 7 weeks later. $A$, Representative examples of Simple Neurite Tracer-generated traces of $\mathrm{TdT}^{+}$neurons in control and NesCreER:caMEK5/TdT mice. $\boldsymbol{B}$, Quantification of number of dendrites in TdT ${ }^{+}$neurons. $\boldsymbol{C}$, Average dendritic length of $\mathrm{TdT}^{+}$neurons. $\boldsymbol{D}$, Sholl analysis of $\mathrm{TdT}^{+}$neurons. $\boldsymbol{E}$, Representative confocal images showing spines of $\mathrm{TdT}^{+}{ }^{+}$neurons. $\boldsymbol{F}$, Quantification of spine density of $\mathrm{TdT}^{+}$neurons. Scale bars, $10 \mu \mathrm{m} . n=4-5$ mice/group; N.S., not significant; ${ }^{*} p<0.05 ;{ }^{* *} p<0.01 ;{ }^{* * *} p<0.001$.

There was a $30 \%$ increase of $\mathrm{BrdU}^{+}$cells in the DG of NesCreER: caMEK5 mice compared with controls $(p=0.038$; Fig. $4 K)$. These data suggest that increased cell survival contributes to caMEK5-induced adult neurogenesis.
We also confirmed that baseline ERK1/2 activity was not altered in the hippocampus of adult mice. Immunohistochemistry analysis with phospho-ERK1/2 (Thr202/Tyr204) antibody showed that the number of phospho-ERK $1 / 2^{+}$cells in the DG 


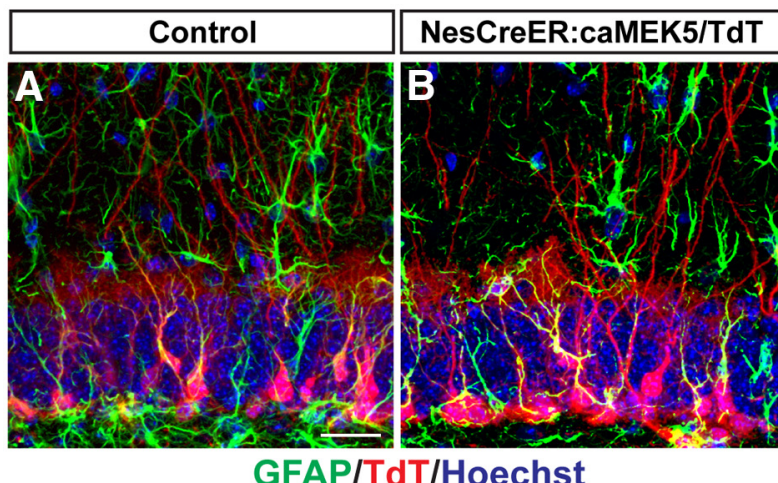

GFAP/TdT/Hoechst
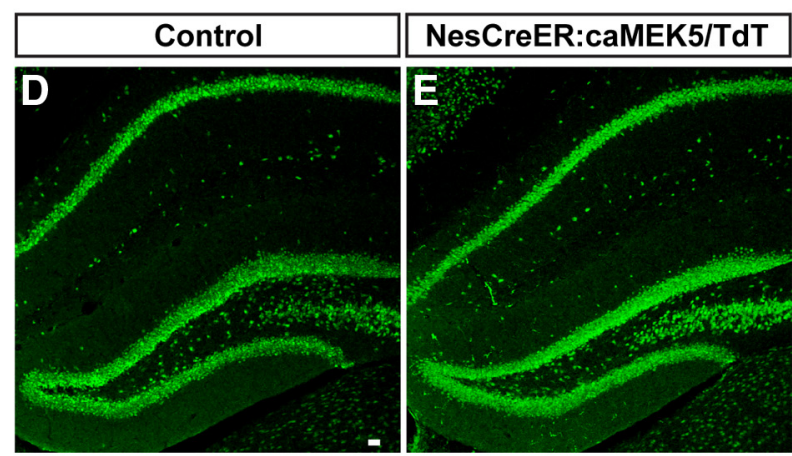

NeuN
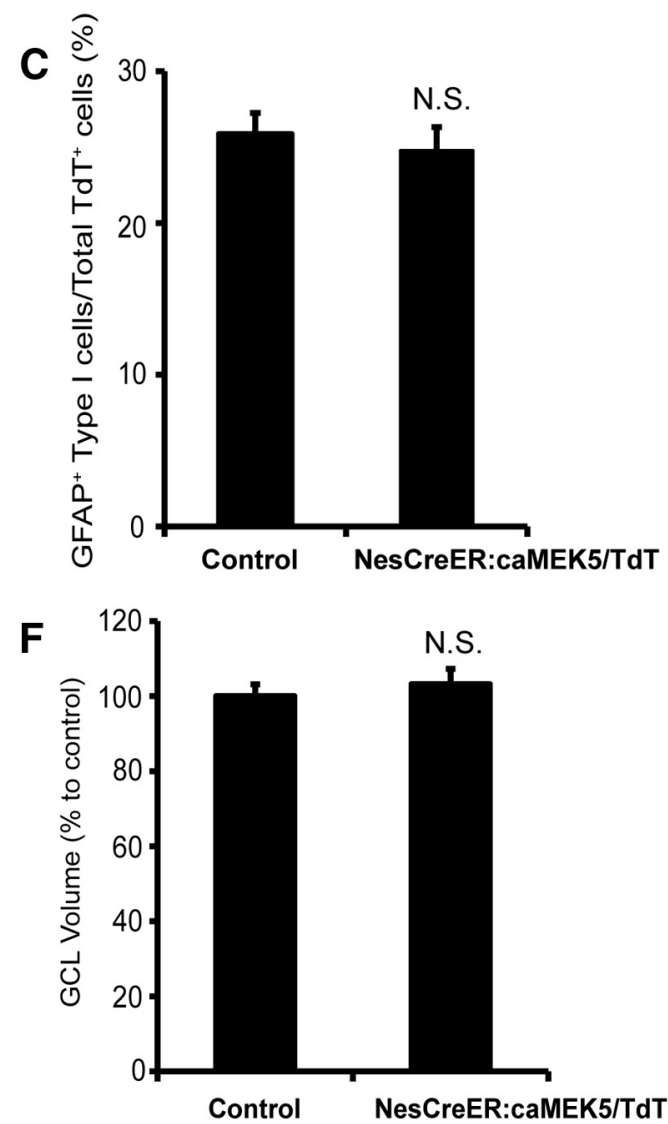

Figure 8. Inducible and conditional caMEK5 activation of ERK5 does not affect adult neural stem cell pool or the volume of the granular cell layer of the DG. $\boldsymbol{A}, \boldsymbol{B}$, Representative GFAP ( $g r e e n$ ) and TdT (red) colabeling from the SGZ of control ( $\boldsymbol{A})$ and NesCreER:caMEK5/TdT (B) mouse brains perfused 7 weeks after the last dose of a $7 \mathrm{~d}$ tamoxifen treatment. Scale bar, $25 \mu \mathrm{m}$. $\boldsymbol{C}$, Quantification of type I radial glia-like aNSC (GFAP ${ }^{+}$, TdT $^{+}$with radial glia-like morphology) in NesCreER:caMEK5/TdT and control mice. $n=4-5$ mice/group. $\boldsymbol{D}, \boldsymbol{E}$, Representative NeuN (green) staining from the SGZ of control (D) and NesCreER:caMEK5/TdT (E) mouse brains perfused 7 weeks after the last dose of a $7 \mathrm{~d}$ tamoxifen treatment. Scale bar, $100 \mu \mathrm{m}$. $\boldsymbol{F}$, Measurement of the granule cell layer volume in NesCreER:caMEK5/TdT and control mice using ImageJ (Sahay et al., 2011). $n=4-5$ mice/group. N.S., not significant.

$(p=0.6$; Fig. $5 A)$ and CA1 pyramidal cell layer $(p=0.79$; Fig. $5 B)$ was comparable between NesCreER:caMEK5 and control mice.

The eGFP tag is only expressed as a caMEK5-eGFP fusion protein in the NesCreER:caMEK5 mice, but not in the littermate controls (Fig. 4A). To compare neuronal differentiation of adultborn cells that have undergone Nestin-CreER-mediated recombination directly in both control and NesCreER:caMEK5 mice, we crossed male Nestin-CreER:ROSA caMEK5-eGFP ${ }^{\text {loxP/+ }}$ mice with a transgenic reporter line, (ROSA26) floxed-stop TdTomato, which expresses the bright fluorescent protein TdTomato (TdT) from the same genomic locus (Gt(ROSA)26Sor; Madisen et al., 2010 ) as the caMEK5-eGFP protein, upon Cre-mediated recombination. Mice treated with tamoxifen of the resulting line, Nestin-CreER:ROSA caMEK5-eGFP ${ }^{\text {loxP }} /$ TdTomato $^{\text {loxP }}$, were designated NesCreER:caMEK5/TdT, whereas similarly treated Nestin-CreER:ROSA TdTomato ${ }^{\text {loxP/+ }}$ littermates, which lack caMEK5-eGFP ${ }^{\text {loxP }}$, were used as controls (Fig. 6A). Mice were treated with tamoxifen for $7 \mathrm{~d}$ and perfused 3 or 7 weeks after the last dose of tamoxifen. We used $\mathrm{TdT}^{+}$as a readout for cells that had undergone Nestin-Cre-mediated recombination in both groups of mice. There was a statistically significant increase in the number of $\mathrm{NeuN}^{+}$neurons among adult-born TdTomato ${ }^{+}$ cells in the DG of NesCreER:caMEK5/TdT mice both at 3 weeks $(p=0.017$; Fig. $6 B-D)$ and 7 weeks $(p=0.0094$; Fig. $6 E-G)$, suggesting that caMEK5 activation promotes neuronal differentiation.
To further confirm that the cellular phenotype we observed is specific to caMEK5 expression, we compared the distribution of $\mathrm{BrdU}^{+}$cells among $\mathrm{TdT}^{+}$versus $\mathrm{TdT}^{-}$cell populations within the same animal. There were an equal number of $\mathrm{BrdU}^{+}$cells in $\mathrm{TdT}^{+}$versus $\mathrm{TdT}^{-}$populations in control mice at 6 weeks after BrdU injection $(p=0.31$; Fig. $6 H-J)$. However, a significantly higher proportion of $\mathrm{BrdU}^{+}$cells were $\mathrm{TdT}^{+}$in NesCreER: caMEK5/TdT mice $(p=0.0012)$, suggesting that there are more adult-born cells in the population that express caMEK5. These data further confirm the specificity of cellular phenotypes observed with caMEK5 mice.

Inducible and conditional activation of ERK5 enhances dendritic complexity of adult-born neurons

We recently found that ERK5 is required for neuronal maturation during adult neurogenesis in the olfactory bulb (Li et al., 2013). We investigated whether ERK5 activation promotes neuronal maturation in the adult DG. NesCreER:caMEK5/TdT and control littermates were killed 7 weeks after the last dose of a $7 \mathrm{~d}$ tamoxifen treatment. $\mathrm{TdT}^{+}$cells in NesCreER:caMEK5/TdT mice had significantly more dendrites than those from controls ( $p=0.00046$; Fig. $7 A, B)$. The average length of all dendrites and the average primary dendritic length of $\mathrm{TdT}^{+}$cells were comparable between the two groups of mice $(p=0.88$ and data not shown; Fig. 7C). Furthermore, $\mathrm{TdT}^{+}$cells from NesCreER: caMEK5/TdT mice had significantly larger numbers of dendritic crossings assessed by Sholl analysis (Fig. 7D). Moreover, Ne- 

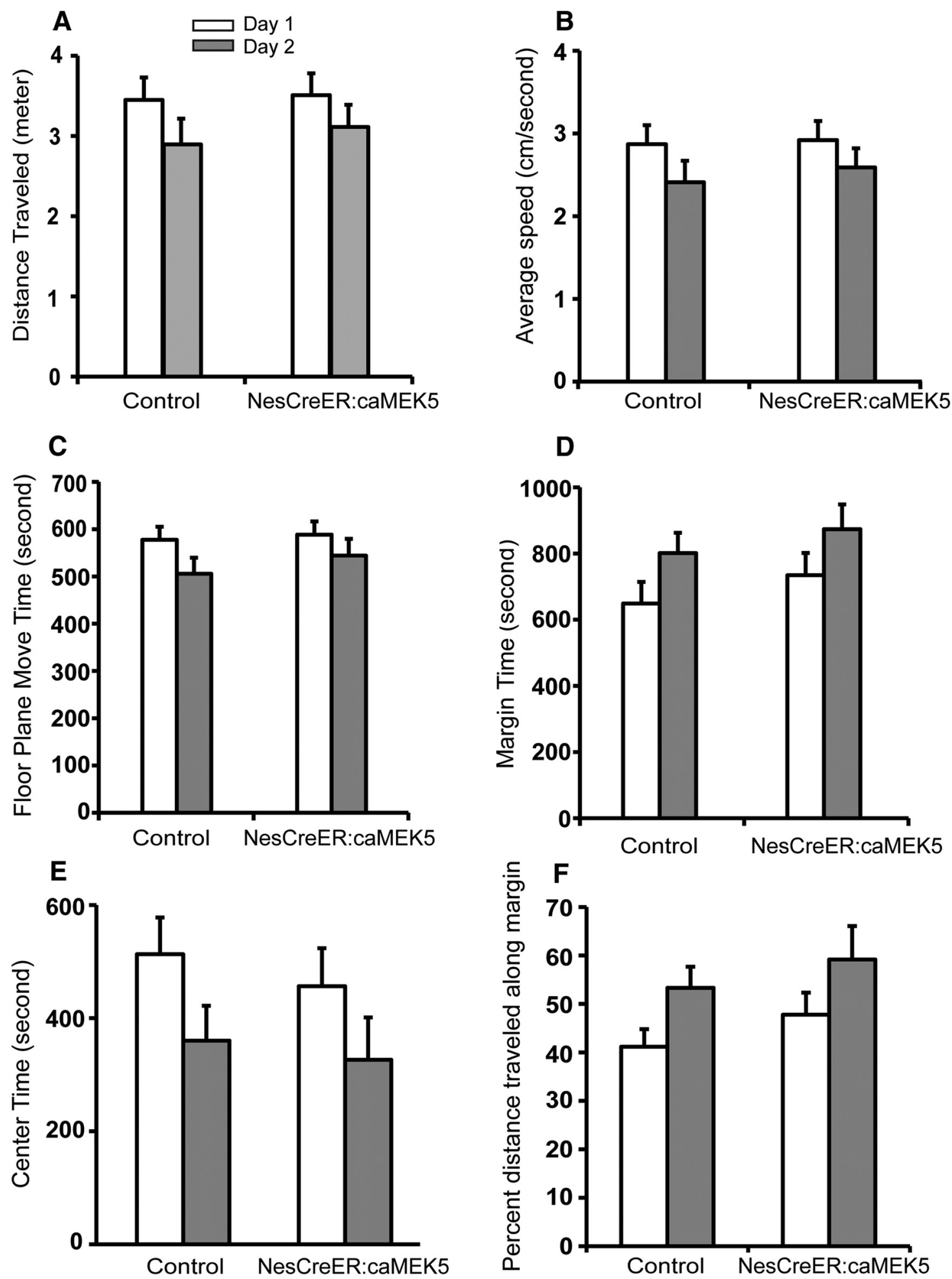

Figure 9. NesCreER:caMEK5 and control mice have comparable basal locomotor activity in the open field tests. NesCreER:caMEK5 and control mice were treated with 3 cycles of a $4 \mathrm{~d}$ tamoxifen regimen with 2 weeks of intercycle interval. Behavior experiments were commenced 2 weeks after the last dose of tamoxifen. Mice were placed in an open field arena for 20 min each day for $2 \mathrm{~d}$. $\boldsymbol{A}$, Total distance traveled on d1 and d2. $\boldsymbol{B}$, Average traveling speed. $\boldsymbol{C}$, Floor plane move time. $\boldsymbol{D}$, Total time spent along the margins of the arena. $\boldsymbol{E}$, Total time spent in the center of the area. $\boldsymbol{F}$, Percent distance traveled along the margin. $n=9-10 /$ group. There was no statistical significant difference between the two groups of mice.

sCreER:caMEK5/TdT mice also have higher spine density $(p<$ 0.001 ; Fig. $7 E, F)$.

We further explored whether caMEK5 activation affects the adult neural stem cell (aNSC) pool. Quantitative analysis of type I radial glia-like aNSCs $\left(\mathrm{GFAP}^{+}, \mathrm{TdT}^{+}\right.$with radial glia-like mor- phology) showed no significant difference between NesCreER: caMEK5/TdT and control mice that were perfused 7 weeks after tamoxifen treatment $(p=0.64$; Fig. $8 A-C)$. Furthermore, the volume of the granule cell layer of the DG at 7 weeks after tamoxifen treatment did not change in NesCreER:caMEK5/TdT mice 

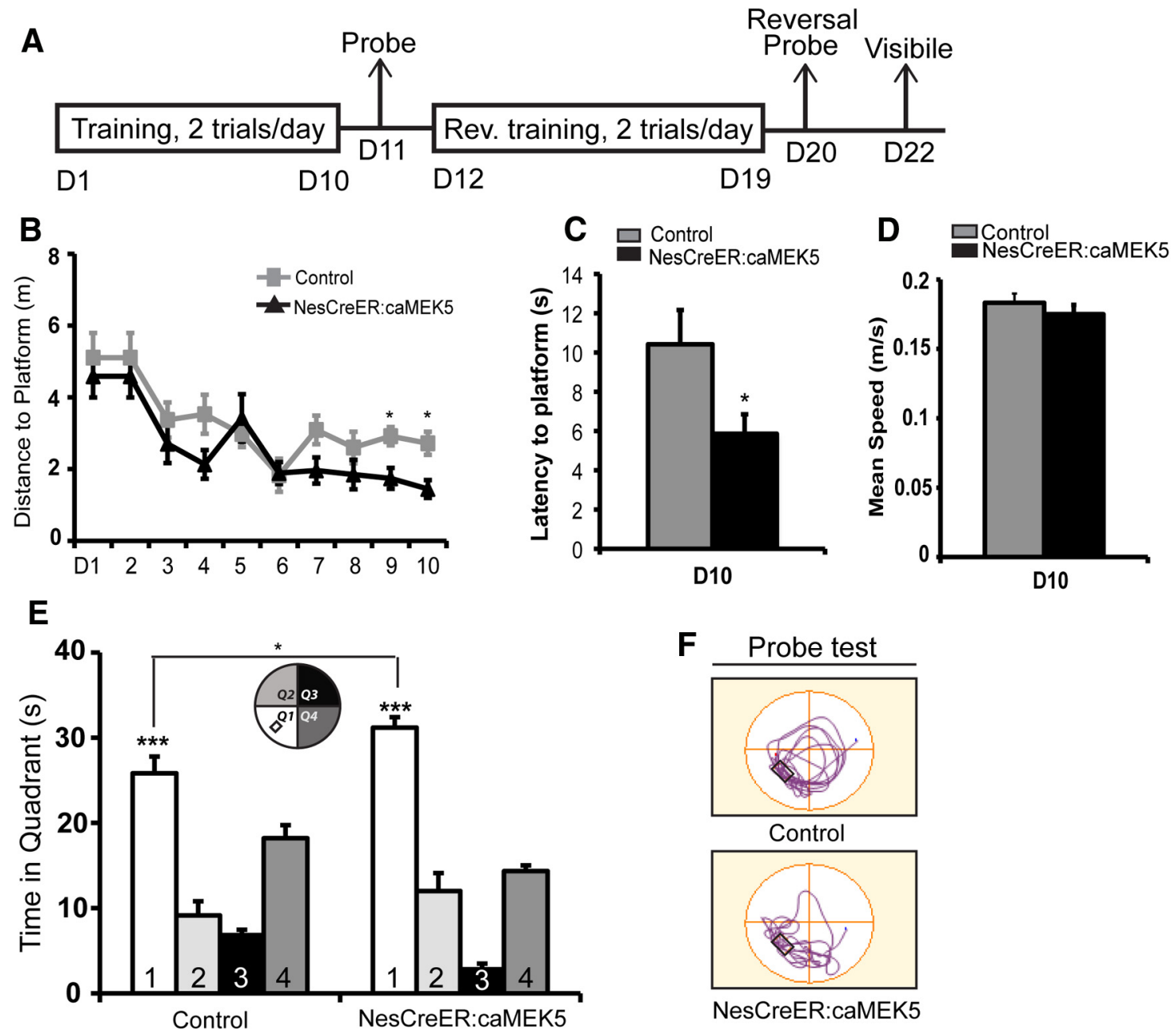

Figure 10. Conditional caMEK5 expression in adult neurogenic regions enhances spatial learning memory formation. $A$, Schematic depiction of the experimental design. Mice were subjected to the hidden platform water maze training ( 2 trials/d for 10 consecutive days, d1-d10), followed by a probe test $1 \mathrm{~d}$ later (d11). Mice were then subjected to reversal training ( 2 trials/d for $8 \mathrm{~d}$, on $\mathrm{d} 12-19$ ) in which the hidden platform was relocated from $Q 1$ to Q3. The reversal probe test was conducted $1 \mathrm{~d}$ after reversal training on $\mathrm{d} 20$ and the visible platform test on $\mathrm{d} 22 . \mathbf{B}$, During the $10 \mathrm{~d}$ training paradigm, NesCreER:caMEK5 mice swam significantly shorter distances than control mice to locate the hidden platform in Q1 on d9 and d 10, suggesting enhanced spatial learning. C, Latency to platform on the last day of training (d10). $\boldsymbol{D}$, The average swim speed quantified on $\mathrm{d} 10 . \boldsymbol{E}$, In the probe test, although both control and NesCreER:caMEK5 mice spent significantly more time in the virtual target quadrant, $Q 1$, than any other three quadrants, NesCreER:caMEK5 mice spent even more time in $Q 1$ than did control mice. One-way ANOVA analysis for control: $F_{(3,36)}=32.7, p<$ 0.001; post hoc analysis, training quadrant (Q1) $>$ all other quadrants, $p<0.001$. One-way ANOVA analysis for Nes(reER:caMEK5: $F_{(3,30)}=48.7, p<0.001 ;$ post hoc analysis, training quadrant (Q1) $>$ all other quadrants, $p<0.001$. $\boldsymbol{F}$, Representative ANYmaze program generated-swimming tracing pattern of control and NesCreER:caMEK5 mice in the probe tests. $n=9-10$ mice/group. ${ }^{*} p<0.05 ;{ }^{* *} p<0.01 ;{ }^{* * *} p<0.001$.

$(p=0.58$; Fig. $8 D-F)$. These data suggest that caMEK5 activation of ERK5 does not affect the adult neural stem cell pool.

\section{NesCreER:caMEK5 mice show normal baseline locomotor activity and no overt anxiety}

To investigate the effect of conditional caMEK5 stimulation of adult neurogenesis on various animal behaviors, we adopted a modified tamoxifen administration regime (Imayoshi et al., 2008; Pan et al., 2012c). Tamoxifen was administered to ROSA caMEK5-eGFP ${ }^{\text {loxP/loxP }}$ and Nestin-CreER ${ }^{\text {TM}}$ :ROSA caMEK5$\mathrm{eGFP}^{\text {loxP/loxP }}$ littermates once a day for $4 \mathrm{~d}$ in each cycle for a total of 3 cycles with 2-week intercycle intervals to obtain control and NesCreER:caMEK5 mice, respectively, for behavior assays. This treatment protocol maximizes Nestin-Cre-mediated recombination for a long period of time without jeopardizing animal survival. We measured the baseline locomotor activity and anxiety level of NesCreER:caMEK5 and control mice for two consecutive days using an open field test. Both mice traveled the same total distance on both days (Fig. 9A) with similar speed (Fig. 9B) and spent similar amounts of time moving around on the floor (Fig. $9 C)$. These data suggest that caMEK5 expression did not affect baseline locomotor activity. Furthermore, both groups of mice spent similar amounts of time along the margins (Fig. 9D) or center area (Fig. 9E) of the open field. Finally, they traveled similar distances along the margins, suggesting that NesCreER: caMEK5 mice do not show increased thigmotaxis (Fig. 9F). The lack of increased thigmotaxis and the fact that they do not spend less time in the center suggest that NesCreER:caMEK5 mice do not show increased anxiety.

NesCreER:caMEK5 mice show improved spatial learning and memory formation

To determine the effect of caMEK5 on the formation of spatial learning and memory, the NesCreER:caMEK5 mice and control littermates were subjected to an MWM test. We adopted a challenging training paradigm with two trials a day rather than four 
A
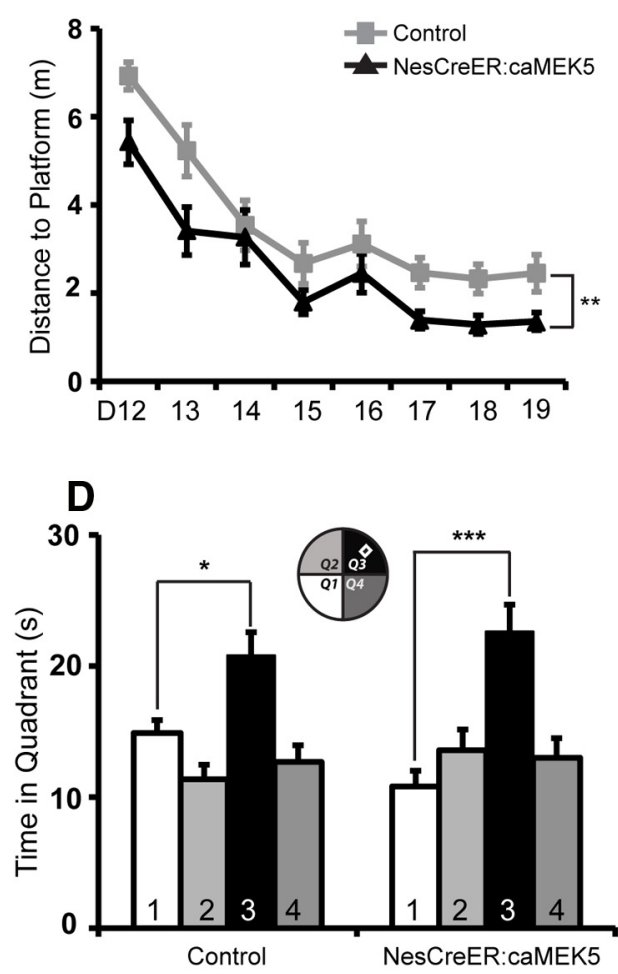

B

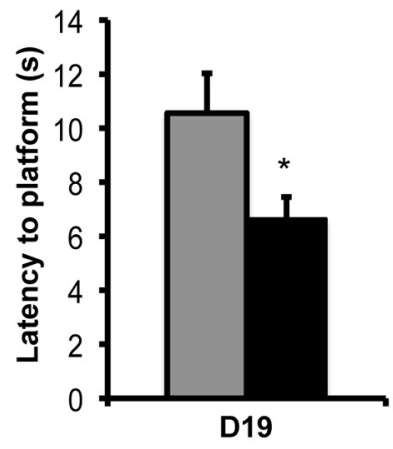

E 口 Control

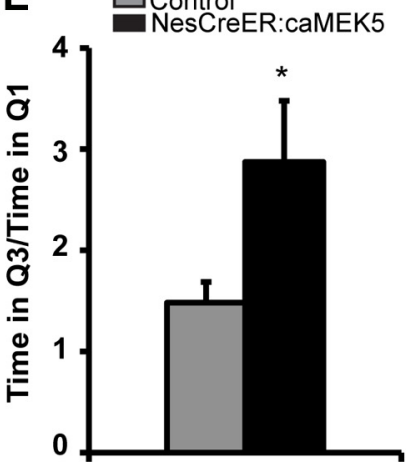

C 口Control

NesCreER:caMEK5

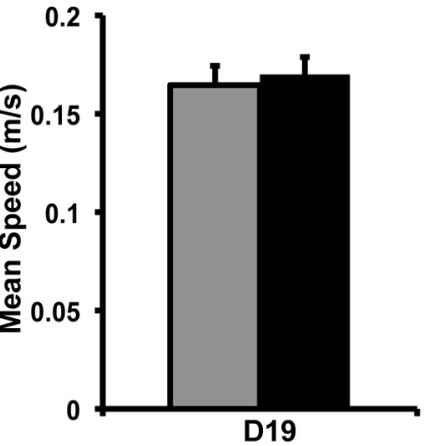

F

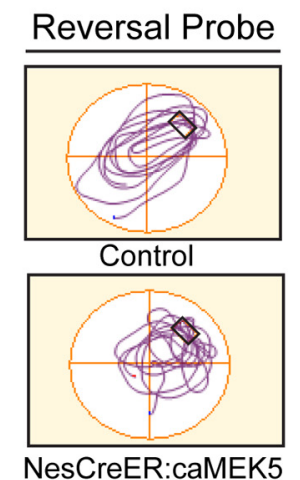

Visible Platform
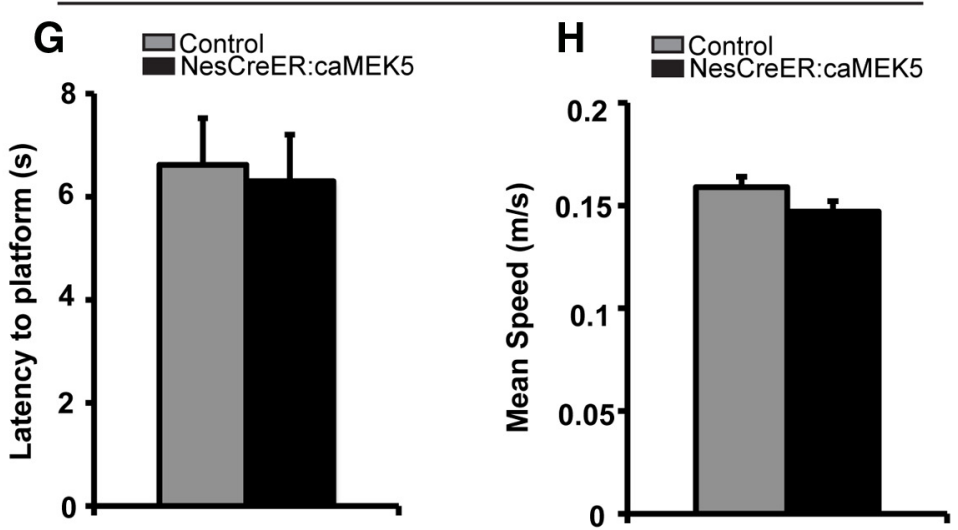

Figure 11. Conditional caMEK5 expression in adult neurogenic regions improves reversal spatial learning memory formation. $A$, Mice were subjected to the reversal hidden platform water maze training after the probe test on $\mathrm{d} 11$, as depicted in Fig. 8A. During the $8 \mathrm{~d}$ reversal training (d12- 119$)$, NesCreER:caMEK5 mice swam shorter distances than control mice to locate the hidden platform in Q3, suggesting enhanced reversal spatial learning. $\boldsymbol{B}$, Analysis of the latency to platform on the last day of reversal training (d19) showed NesCreER:caMEK5 mice spent less time than control mice to find the hidden platform. $C$, The average swim speed on d19 training was comparable between the two groups of mice. $\boldsymbol{D}$, In the reversal probe test, both control and NesCreER:caMEK5 mice spent significantly more time in the new virtual target quadrant Q3 than in the old target quadrant (Q1). $E$, The ratio of time spent in Q3 versus Q1 in reversal probe test was significantly higher for NesCreER:caMEK5 mice than control mice. $\boldsymbol{F}$, Representative ANYmaze program generated-swimming tracing pattern of control and NesCreER:caMEK5 mice in the reversal probe tests. $\boldsymbol{G}, \boldsymbol{H}$, Control and NesCreER:caMEK5 mice performed equally well in the visible platform test; they showed comparable latency to acquisition of the platform location ( $\boldsymbol{G}$ ) and similar mean swimming speed $(\boldsymbol{H})$. $n=9-10$ mice/group. ${ }^{*} p<0.05 ;{ }^{* *} p<0.01 ; * * p<0.001$.

trials a day in this assay. Mice were trained for 10 consecutive days (d1-d10) in the MWM test in which the hidden platform was placed in the same location (Q1; Fig. 10A).

Both NesCreER:caMEK5 and control mice learned the task over this $10 \mathrm{~d}$ training period, manifested as swimming shorter distances to find the hidden platform (Fig. 10B). Interestingly, NesCreER:caMEK5 mice swam significantly shorter distances than control mice to find the hidden platform on $\mathrm{d} 9$ and $\mathrm{d} 10$ $(p<0.05)$. Analysis of the latency to platform on the last day of training (d10) also showed that NesCreER:caMEK5 mice needed less time than control mice to find the hidden platform $(p=0.039$; Fig. $10 C)$, although the average swim speed was similar between the two groups of mice (Fig. 10D). In the probe test $1 \mathrm{~d}$ after training (d11), both groups spent much more time in the target Q1 searching the hidden platform than in all other quadrants (Fig. 10E,F), but NesCreER:caMEK5 mice spent even more time in Q1 than did control mice ( $p=$ 0.04; Fig. 10E).

These mice were then subjected to a reversal training paradigm in which the hidden platform was moved from Q1 to the 
A
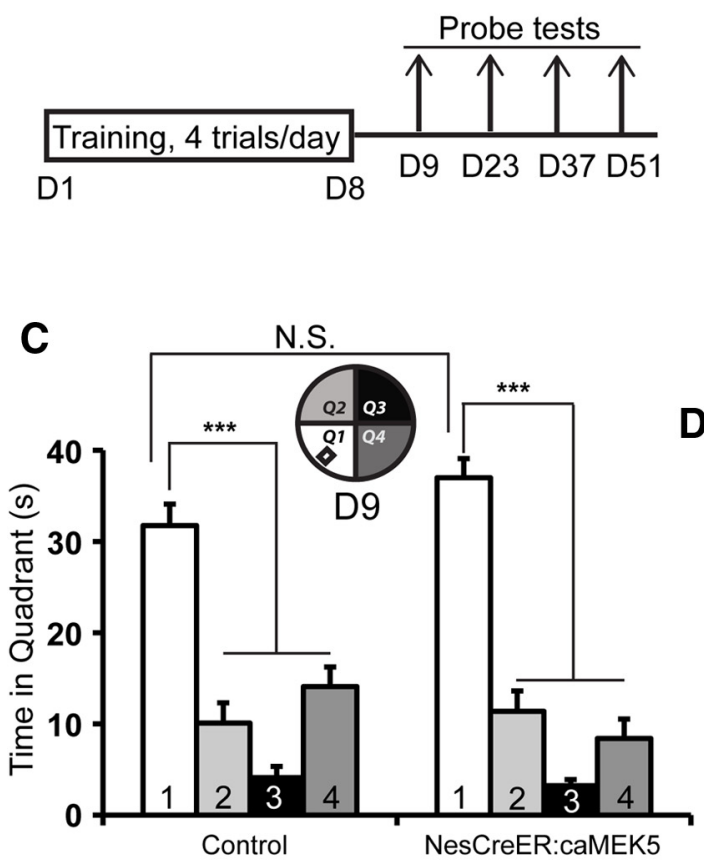

E

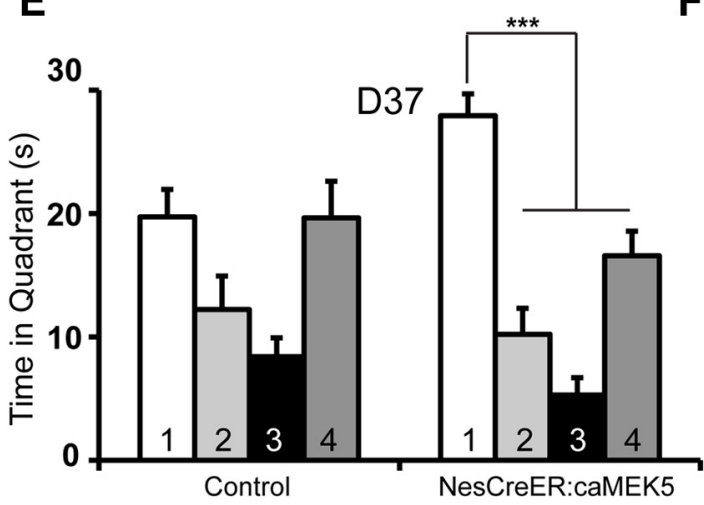

$\mathbf{F}$
B

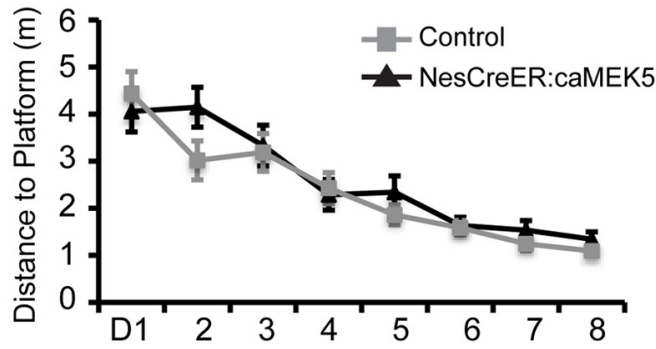

G
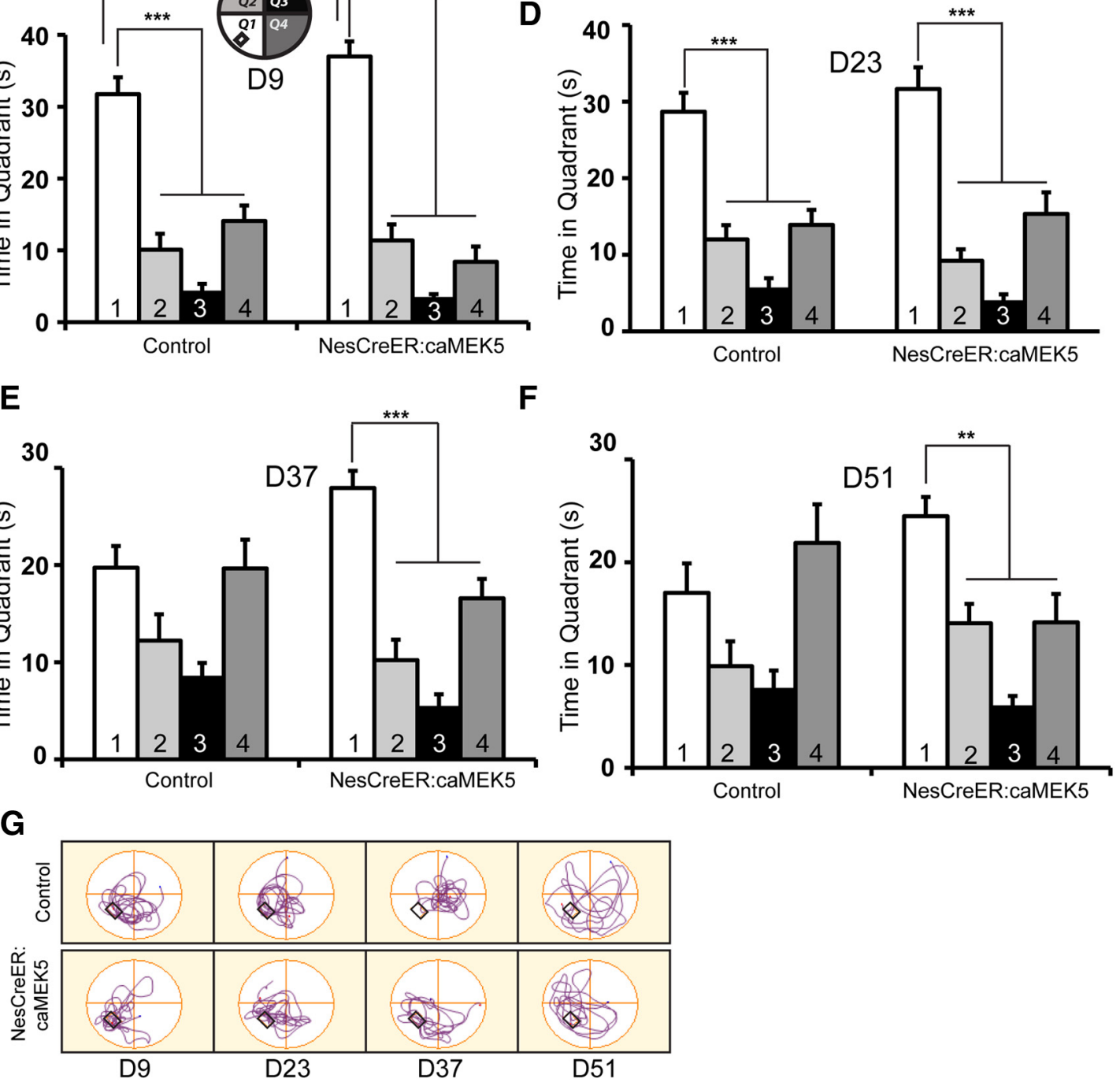

Figure 12. Conditional caMEK5 expression in adult neurogenic regions extends long-term spatial memory. $A$, Schematic depiction of the experimental design. Mice were subjected to training for $8 \mathrm{~d}(\mathrm{~d} 1-\mathrm{d} 8)$ with 4 trials/din the hidden platform water maze. The first probe test was conducted on $\mathrm{d} 9$, the day after the last day of training, and second, third, and fourth probe tests were conducted every 2 weeks on $\mathrm{d} 23$, d37, and d51, respectively. $\boldsymbol{B}$, During the $8 \mathrm{~d}$ training paradigm, target acquisition of the platform located in $\mathrm{Q1}$ was similar for control and NesCreER:caMEK5 mice. $\boldsymbol{C}$, Both control and NesCreER:caMEK5 mice spent significantly more time in $Q 1$ than any other quadrants in the first probe test on d9. One-way ANOVA analysis for control: $F_{(3,32)}=34.0, p<0.001 ; p 0 s t$ hoc analysis, time in training quadrant (01) was more than that of any other quadrants, $p<0.001$. One-way ANOVA analysis for NesCreER:caMEK5 mice: $F_{(3,28)}=62.5, p<0.001 ; p 0 s t h o c$ analysis, time in training quadrant ( $Q 1)$ was more than that of any other quadrants, $p<0.001$. There is no statistically significant difference in time spent in $Q 1$ between the two groups of mice $(p=0.12)$, suggesting that the two groups learned equally well. $\boldsymbol{D}$, In the second probe test conducted on $\mathrm{d} 23$, both control and NesCreER:caMEK5 mice still remembered $\mathrm{Q} 1$ and spent significantly more time in $Q 1$ than any other quadrants. One-way ANOVA analysis for control: $F_{(3,32)}=28.2, p<0.001$; post hoc analysis, time in training quadrant (01) was more than that of any other quadrants, $p<$ 0.001. One-way ANOVA analysis for NesCreER:caMEK5 mice: $F_{(3,28)}=30.3, p<0.001$; post hoc analysis, time in training quadrant (Q1) was more than that of any other quadrants, $p<0.001$. $E$, During the third probe test on $\mathrm{d} 37$, NesCreER:caMEK5, but not control mice, spent significantly more time in $Q 1$ than any other quadrants. One-way ANOVA analysis for NesCreER:caMEK5: $F_{(3,28)}=$ $31.3, p<0.001$; post hoc analysis, time in training quadrant (Q1) was more than that of any other quadrants, $p<0.001$. One-way ANOVA analysis for control mice: $F_{(3.32)}=5.4, p=0.004 ; p 0 s t$ hoc analysis, time in $\mathbf{Q 1}$ was only more than that of the opposite quadrant (Q3), $p<0.01$. $F$, NesCreER:caMEK5 mice, but not control mice, retained spatial memory for $Q 1$ even at 6 weeks after training when the fourth probe test was conducted on d51. One-way ANOVA analysis for NesCreER:caMEK5 mice: $F_{(3,28)}=11.2, p<0.001$; post hoc analysis, time in $Q 1$ was more than that of any other quadrants, $p<0.01$. One-way ANOVA analysis for control mice: $F_{(3,32)}=7.1, p=0.001$; post hoc analysis, time in $Q 1$ was only more than that of $Q 3, p<0.05$. G, Representative swimming pattern traces in the four probe tests generated by the ANYmaze program. $n=8-9$ mice/group. ${ }^{*} p<0.05 ;{ }^{* *} p<0.01 ;{ }^{* * *} p<0.001$. 
A
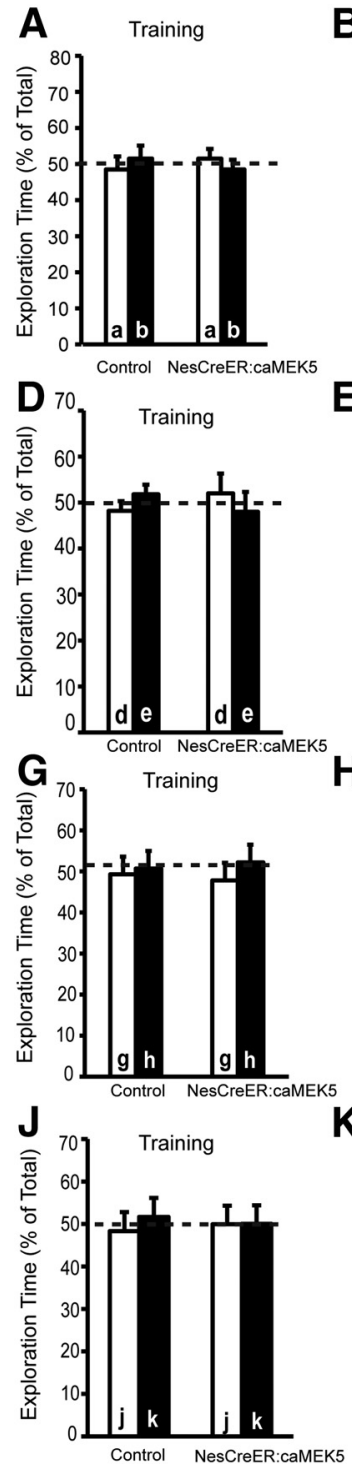

B
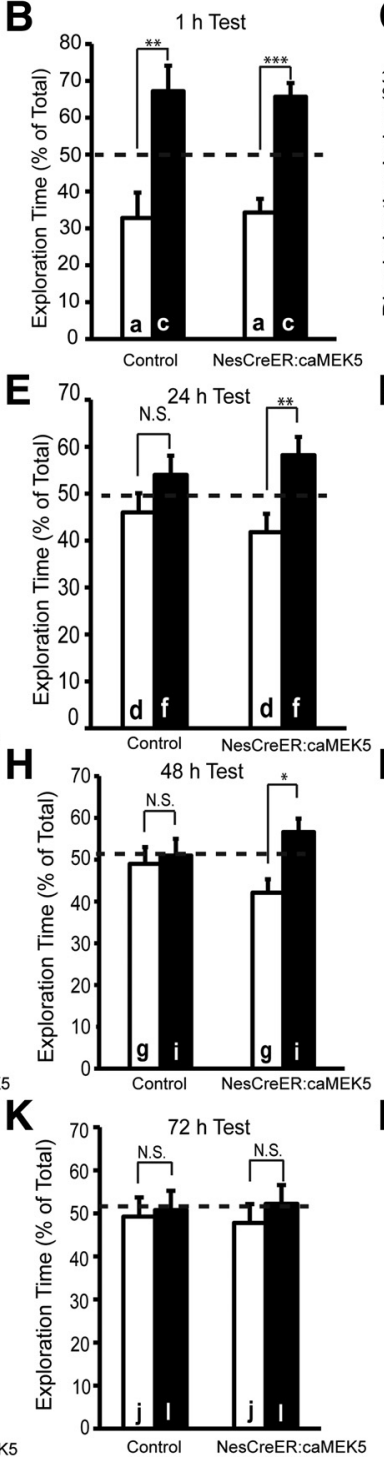

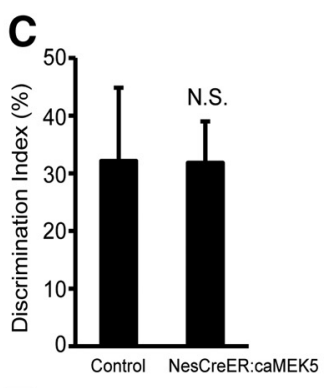

$\mathbf{F}$
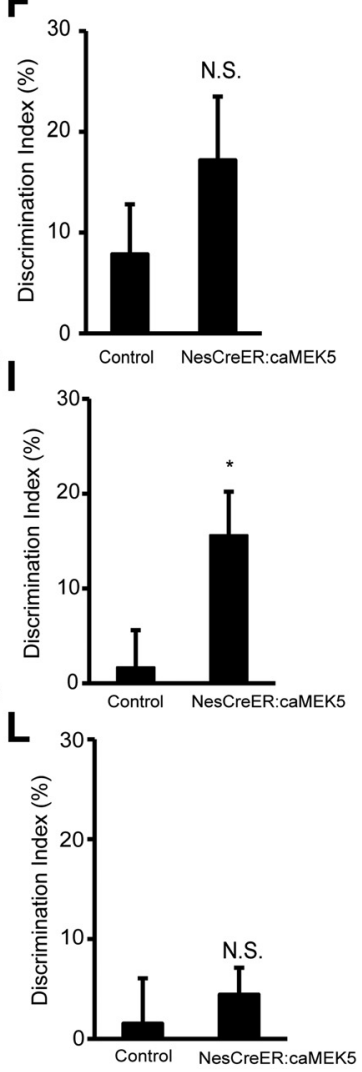

Figure 13. NesCreER:caMEK5 mice have longer memory in the novel object recognition test. Mice were trained with 2 objects for $5 \mathrm{~min}$ and the percentage of time spent on exploring each object was quantified. Mice were then introduced to 2 objects at 1 , 24,48 or $72 \mathrm{~h}$ later, one familiar (object a, $\mathrm{d}, \mathrm{g}$, or j) and one novel (object $c, \mathrm{f}, \mathrm{i}$, or l) as labeled in the figure, and the percentage of time spent on each object during a 5 min test period and the discrimination index was quantified. $\boldsymbol{A}-\boldsymbol{C}$, Training and $1 \mathrm{~h}$ test. $\boldsymbol{D}-\boldsymbol{F}$, Training and $24 \mathrm{~h}$ test. G-I, Training and $48 \mathrm{~h}$ test. $\boldsymbol{J}-\boldsymbol{L}$, Training and $72 \mathrm{~h}$ test. $n=9-10 /$ group. N.S., not significant; ${ }^{*} p<0.05$; ${ }^{* *} p<0.01 ;{ }^{* * *} p<0.001$.

opposite location, Q3, and mice were trained twice a day for 8 consecutive days from d12-d19. Similar to initial training, NesCreER:caMEK5 mice swam shorter distances than control mice to locate the hidden platform during this reversal training period (ANOVA with repeated measures $F_{(1,17)}=11.2, p=0.004$; Fig. $11 A)$. Data from the reversal training collected on d19 further showed that the latency for NesCreER:caMEK5 mice to find the hidden platform was shorter than that for control mice ( $p=0.03$; Fig. 11B), whereas the average swim speed was comparable (Fig. 11C). Furthermore, although both groups of mice spent more time in the new target quadrant $(\mathrm{Q} 3)$ than in the old one $(\mathrm{Q} 1)$ in the subsequent reversal probe test on $\mathrm{d} 20$ ( $t$ test, control, $p=$ 0.013; NesCreER:caMEK5, $p=0.0002$; Fig. $11 D, F)$, the ratio of time spent in Q3 over that in Q1 was higher for NesCreER: caMEK5 mice (2.8-fold) than control mice (1.5-fold, $p=0.04$; Fig. 11E). Two days after the reversal probe test, mice were subjected to a visible platform test in which both groups of mice had

similar latency to platform ( $p=0.79$; Fig. $11 G)$ and mean swim speed ( $p=0.21$; Fig. $11 \mathrm{H})$. Therefore, the differences we observed in the hidden platform training and tests were not caused by differences in vision, ability to swim, or motivation to escape. These data suggest that NesCreER: caMEK5 mice exhibit enhanced acquisition of spatial learning and memory formation.

\section{NesCreER:caMEK5 mice have longer hippocampus-dependent memory}

To determine whether NesCreER:caMEK5 mice also have extended long-term spatial memory, a separate cohort of mice were trained in the hidden platform of the MWM using a commonly used training paradigm in which mice were trained for four trials a day until the learning curve reached a plateau (Fig. 12A; Deng et al., 2009; Pan et al., 2012c). This more intense (thus easier) training paradigm was used so both groups of mice learned the task equally well, thus allowing a clear evaluation of subsequent long-term memory retention (ANOVA with repeated measures, $F_{(1,15)}=1.058, p=$ 0.32 ; Fig. $12 B)$. In the probe test performed $1 \mathrm{~d}$ after the last day of training (d9), both mice performed equally well; they spent much more time in the target quadrant (Q1) compared with all other three quadrants (Fig. 12C, G, see figure legends for oneway ANOVA analysis). Furthermore, there was no statistically significant difference in time in Q1 between control and NesCreER: caMEK5 mice. These mice were subjected to the probe test again on $\mathrm{d} 23, \mathrm{~d} 37$, and $\mathrm{d} 51$ to evaluate long-term spatial memory retention. Although both control and NesCreER: caMEK5 mice spent more time in the target quadrant (Q1) compared with all other three quadrants when tested on $\mathrm{d} 23$ (Fig. $12 D, G$, see figure legends for one-way ANOVA analysis), only NesCreER: caMEK5 mice retained strong spatial memory on $\mathrm{d} 37$ and $\mathrm{d} 51$ and searched target Q1 preferentially over any of the other three quadrants (Fig. $12 E-G)$

The effect of genetic enhancement of adult neurogenesis by caMEK5 on long-term memory was further ascertained by subjecting animals to a novel object recognition test, which is at least partly dependent on hippocampal function and adult neurogenesis (Shimizu et al., 2007; Pan et al., 2012c). Both control and NesCreER:caMEK5 mice remembered the familiar object "a" equally well $1 \mathrm{~h}$ after training and spent significantly more time investigating the novel object " $c$ " $(p=0.0023$ for control and $p=$ 0.00017 for NesCreER:caMEK5; Fig. 13 $A, B$ ). When the memory for the familiar object was tested $24 \mathrm{~h}$ after training, NesCreER: caMEK5 mice spent significantly more time investigating the novel object " $\mathrm{f}$ " than the familiar object " $\mathrm{d}$ " $(p=0.0096$; Fig. $13 D, E$ ), indicating that they remembered the familiar object. Although control mice spent slightly more time investigating the novel object "f" than the familiar object "d," the difference was 
not statistically significant ( $p=0.18)$. By $48 \mathrm{~h}$, control mice showed no preference for either object $(p=0.72)$, whereas NesCreER:caMEK5 mice still retained memory for the familiar object "g" and spent significantly more time investigating the novel object "i" ( $p=0.011$; Fig. 13G,H). The discrimination index, defined as the difference between exploration times for the novel and familiar objects divided by total exploration time (Haettig et al., 2011), was significantly higher in NesCreER:caMEK5 mice than controls at $48 \mathrm{~h}$ $(p=0.034$; Fig. 13I). By 72 h, neither groups showed preference for either object (Fig. 13J-L).

We also subjected the mice to an object novel location test, another hippocampus-dependent spatial learning and memory task (Oliveira et al., 2010). Two identical objects were used in training; one of them was moved to a novel location during tests conducted 1,48 , or $72 \mathrm{~h}$ after training. We added a second control group of mice, Nestin-CreER ${ }^{\mathrm{TM}}$ :ROSA caMEK5-eGFP ${ }^{\text {loxP/loxP }}$ littermates treated with vehicle control (Fig. 14A) in this experiment to address any concern that the gain-of-function phenotype we observed with the NesCreER:caMEK5 mice may be due to the presence of Nestin-CreER ${ }^{\mathrm{TM}}$. NesCreER:caMEK5 mice and both groups of controls remembered the familiar location "a" equally well $1 \mathrm{~h}$ after training and spent significantly more time investigating the object at the novel location " $\mathrm{c}$ " $(p<0.001$ for controls, and $p<0.0001$ for NesCreER:caMEK5; Fig. 14B,C). There was no difference in the discrimination indexes among the different groups of mice (Fig. 14D). By $48 \mathrm{~h}$, however, only the NesCreER:caMEK5 mice retained memory for the familiar location $(p<$ 0.001 for NesCreER:caMEK5; Fig. $14 E, F$ ) and had a significantly higher discrimination index than the two control groups ( $p=0.04$ for control vs NesCreER: caMEK5 and $p=0.03$ for vehicle vs NesCreER:caMEK5; Fig. $14 G)$. By $72 \mathrm{~h}$, none of the mice showed memory for object location (Fig. 14H-J). Together, the data shown in Figures 12, 13, and 14 suggest that NesCreER:caMEK5 mice have superior hippocampus-dependent long-term memory compared with control littermates.

\section{Discussion}

Learning and memory decline with aging and are affected by neurological diseases including epilepsy, depression, Alzheimer's disease, and Parkinson's disease. Adult hippocampal neurogenesis has been implicated in several forms of hippocampusdependent memory and is adversely affected by aging and neurodegenerative diseases (Zhao et al., 2008). Therefore, there is considerable interest in developing strategies to improve learning and memory persistence and to identify molecular mechanisms
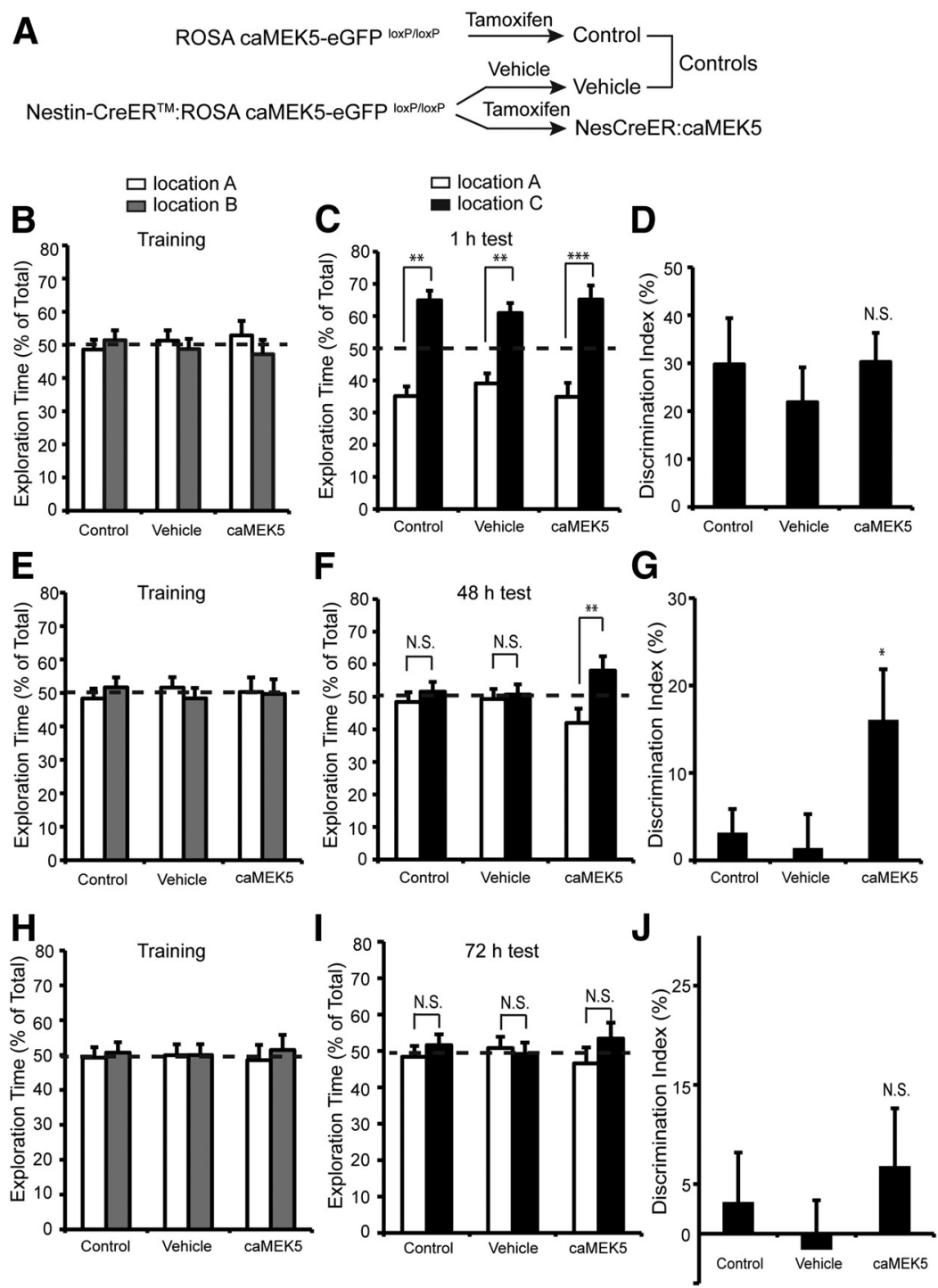

Figure 14. NesCreER:caMEK5 mice have longer memory in the object location recognition test. $A$, Tamoxifen treatment scheme for generating NesCreER:caMEK5 and two groups of control mice from littermates. $\boldsymbol{B}$, Mice were trained with two identical objects located at difference places (location a and b) for 5 min and the percentage of time spent on exploring each object was quantified. At 1,48 , or $72 \mathrm{~h}$ after training, mice were introduced to the same two objects where one remained at the same location (location the discrimination index were quantified. $\boldsymbol{B}-\boldsymbol{D}$, Training and $1 \mathrm{~h}$ test. $\boldsymbol{E}-\mathbf{G}$, Training and $48 \mathrm{~h}$ test. $\boldsymbol{H}-\boldsymbol{J}$, Training and $72 \mathrm{~h}$ test. $n=$ $10-11 /$ group. N.S., not significant; ${ }^{*} p<0.05 ;{ }^{* *} p<0.01 ;{ }^{* * *} p<0.001$.

that regulate adult neurogenesis. Identification of endogenous signaling molecules that can be manipulated to stimulate adult neurogenesis may aid the development of therapeutic strategies to correct cognitive disorders. The goal of this study was to develop a transgenic mouse model to activate endogenous ERK5 selectively in adult neurogenic regions and to determine whether this genetic gain of function is sufficient to stimulate adult neurogenesis, improve learning, and increase memory persistence.

We generated a line of knock-in mice in which endogenous ERK5 in adult neural stem cells can be conditionally activated by tamoxifen-induced expression of a constitutive active MEK5 through Nestin-CreER ${ }^{\mathrm{TM}}$-mediated recombination. We confirmed biochemically that the expression of caMEK5-eGFP fusion protein in the brain is tightly controlled by Cre-mediated recombination and, when expressed, caMEK5-eGFP specifically activates endogenous ERK5, but not the closely related ERK1/2. It 
is important to note that the caMEK5-eGFP fusion protein was not overexpressed; rather, it was expressed at a level similar to that of the endogenous MEK5 protein. Evidence from two strains of knock-in mice, NesCreER:caMEK5 and NesCreER:caMEK5/ TdT mice, demonstrated that expression of caMEK5 increases the number of adult-born neurons in the SGZ by promoting cell survival without affecting cell proliferation or the stem cell pool. Furthermore, caMEK5 expression promotes neuronal differentiation, evidenced by increases in the number of adult born $\mathrm{DCX}^{+}$ and $\mathrm{NeuN}^{+}$cells and the number of dendrites, spine densities, and dendritic crossings. Therefore, inducible and targeted ERK5 activation was sufficient to stimulate adult neurogenesis in the hippocampus.

Although a number of studies have investigated the functional consequence of suppressing adult neurogenesis on learning and memory, only a few reports have investigated whether cognition can be improved by increasing adult neurogenesis. Animals subjected to an enriched environment (Kempermann et al., 1997; Birch et al., 2013), voluntary running (van Praag et al., 1999; Creer et al., 2010), and chronic -antidepressant treatment (David et al., 2009) showed increased adult neurogenesis and improved memory formation. More recently, Petrik et al. discovered that Isx-9, a synthetic small molecule, increases hippocampal neurogenesis and enhances memory in the MWM probe test (Petrik et al., 2012). Wang et al. reported that metformin, a widely used drug for treating type 2 diabetes, promotes adult neurogenesis through an atypical PKC-CBP pathway and enhances reversal spatial learning in the MWM test (Wang et al., 2012). These studies generated much excitement about the possibility that enhancing adult neurogenesis may improve memory formation.

Additional studies using more selective approaches, such as conditional and inducible gene manipulation to specifically induce adult neurogenesis without affecting other biochemical or biological pathways, are needed to establish a direct link between increased adult neurogenesis and cognition. To date, only one such genetic study has been reported. Sahay et al. generated a transgenic mouse line to delete the proapoptotic gene Bax specifically in adult neural stem cells; as a result, more of the adult-born neurons survived (Sahay et al., 2011). These mice performed better than control mice at distinguishing between similar contexts in a fear-conditioning test, suggesting that increased adult hippocampal neurogenesis improves pattern separation.

The data presented in this study provide strong and clear genetic evidence supporting a causal relationship between enhanced adult neurogenesis and improved spatial memory formation. When trained 4 times a day for $8 \mathrm{~d}$, both NesCreER: caMEK5 and control mice learned equally well in the hidden platform MWM test. At the end of training, both groups of mice swam about $1.2 \mathrm{~m}$ before they found the hidden platform. When they were trained only twice a day, the learning curve for both mice plateaued after $7 \mathrm{~d}$ of training; control and NesCreER: caMEK5 mice swam about 2.7 and $1.4 \mathrm{~m}$, respectively, before they located the hidden platform. Because there was no difference observed in swim speed or latency to escape water in the visible platform test, the difference observed here (2.7 vs $1.4 \mathrm{~m})$ is not due to variable swimming ability or motivation to escape water. Therefore, this less intensive training paradigm is more challenging to mice and NesCreER:caMEK5 mice learned better than controls under this condition. NesCreER:caMEK5 mice also learned better in the reversal training and had stronger memory than control mice in both the initial probe test and reversal probe test. These data suggest that increasing adult neurogenesis is suf- ficient to improve learning acquisition and memory formation for more demanding tasks.

There have been no studies reporting whether increasing adult neurogenesis can extend the duration of memory. We present evidence that, although both NesCreER:caMEK5 and control mice learned equally well during training and formed similar degrees of initial memory after training 4 times a day for $8 \mathrm{~d}$ in the hidden platform MWM, the duration of long-term spatial memory was extended at least 3 times, from 2 to 6 weeks, by conditional ERK5 activation. In the novel object recognition test and the novel location test, the memory for novel objects or novel locations was extended from 1 to $48 \mathrm{~h}$ by caMEK5 expression. Collectively, these data provide the first evidence that selective and conditional enhancement of adult neurogenesis can significantly delay memory decay and extend the duration of long term, hippocampus-dependent memory.

In addition to its effect on the hippocampus, expression of caMEK5 also enhances adult neurogenesis in the SVZ (data not shown). Although we could not completely rule out the possibility, we think it highly unlikely that the improved cognitive function observed with the NesCreER:caMEK5 mice is due to a changes in SVZ adult neurogenesis. The behavioral assays used in this study to assess cognition, including the MWM and novel object location tests, are hippocampus-dependent tasks (Deng et al., 2010). Furthermore, we have strictly eliminated any potential olfactory cues in these assays. For example, in the novel object recognition or object novel location assays, the objects and testing arena were extensively cleaned with $5 \%$ acetic acid after each use.

Signaling mechanisms underlying adult neurogenesis are not well defined. We previously demonstrated that, in the adult brain, ERK5 expression is specific to the neurogenic regions and generally absent in the rest of the brain. Conditional loss of ERK5 expression selectively in the adult neurogenic regions inhibits SGZ adult neurogenesis and impairs several forms of hippocampus-dependent memory formation, including spatial learning (Pan et al., 2012a; Pan et al., 2012c; Pan et al., 2012d). Furthermore, viral expression of a constitutive active MEK5, an upstream-activating kinase for ERK5, stimulates adult neurogenesis both in vitro and in vivo (Pan et al., 2012c; Pan et al., 2012d). Together with data presented here, our results suggest that activation of ERK5 is both necessary and sufficient to promote adult neurogenesis in vivo and increase hippocampusdependent memory formation. MEK5-ERK5 is an endogenous MAP kinase signaling pathway that can be activated by extracellular signals present in the hippocampus that are known to promote adult neurogenesis, including neurotrophins (Pan et al., 2012d). Our data provide the first evidence that direct activation of a single endogenous signaling pathway selectively in adult neurogenic regions is sufficient to enhance adult neurogenesis in the hippocampus and improve hippocampus-dependent memory formation.

How adult-born neurons in the hippocampus contribute to memory persistence is unknown and remains an interesting question to be addressed in the future. Memory consolidation and maintenance depends on de novo transcription and protein synthesis (Bekinschtein et al., 2007), including repeated rounds of NMDA receptor synthesis (Cui et al., 2004), suggesting that memory persistence requires recurrent rounds of consolidation. The cAMP/MAPK/CREB pathway, which is important for memory consolidation, is activated during REM sleep during the circadian cycle (Eckel-Mahan et al., 2008; Luo et al., 2013). This reactivation of the memory consolidation pathway during REM 
sleep may generate repeated rounds of transcription and translation required for the persistence of the memory trace. There is also electrophysiological evidence that the same sequence of synaptic firing that occurs during training for a spatial memory task is repeated during sleep (Lee and Wilson, 2002). It is possible that, during the repeated synaptic firing in sleep, new synapses, including those from adult-born neurons, are recruited to the circuitry for continued maintenance of established memories. For example, immature adult-born neurons have different activation threshold than mature granule neurons in the DG; a significant proportion of the immature adult born neurons, but not mature neurons, are activated by weak afferent activity (MarínBurgin et al., 2012). Because most, if not all, memory traces eventually decay, it is tempting to speculate that the strength of recurrent synaptic firing encoding a specific memory trace may gradually reduce over time. Because of their higher excitability in response to weak stimulations, immature adult-born neurons may be more suited for new synapse recruitment during recurrent synaptic firing in sleep, especially as time goes on and the memory trace weakens. When adult neurogenesis is impaired, fewer adult-born neurons and their synapses will be available to respond to the gradually weakening synaptic firing and be recruited to the memory circuitry, thus accelerating memory decay and shortening long-term memory. In contrast, enhancing adult neurogenesis by increasing the pool of immature adult born neurons and/or the number of dendrites and spines will likely expand the number of responding synapses, strengthen the memory circuitry, and thus extend long-term memory.

In summary, we developed an inducible genetic approach to selectively enhance adult neurogenesis through specific activation of endogenous ERK5 MAP kinase. Our data demonstrate that activation of a single signaling pathway, that of the ERK5 MAP kinase, is sufficient to enhance adult neurogenesis and improve spatial learning under challenging conditions and to significantly extend the duration of memory. The discovery that memory can be prolonged by stimulating adult neurogenesis has important implications for the development of therapeutic strategies to treat memory disorders. The unique expression pattern of ERK5 in the adult brain and its demonstrated role in both regulation and promotion of adult neurogenesis and memory retention provides a pharmacological window of opportunity to improve the duration of long-term memory.

\section{References}

Bekinschtein P, Cammarota M, Igaz LM, Bevilaqua LR, Izquierdo I, Medina $\mathrm{JH}$ (2007) Persistence of long-term memory storage requires a late protein synthesis- and BDNF-dependent phase in the hippocampus. Neuron 53:261-277. CrossRef Medline

Birch AM, McGarry NB, Kelly AM (2013) Short-term environmental enrichment, in the absence of exercise, improves memory, and increases NGF concentration, early neuronal survival, and synaptogenesis in the dentate gyrus in a time-dependent manner. Hippocampus 23:437-450. CrossRef Medline

Cavanaugh JE (2004) Role of extracellular signal regulated kinase 5 in neuronal survival. Eur J Biochem 271:2056-2059. CrossRef Medline

Cavanaugh JE, Ham J, Hetman M, Poser S, Yan C, Xia Z (2001) Differential regulation of mitogen-activated protein kinases ERK1/2 and ERK5 by neurotrophins, neuronal activity, and cAMP in neurons. J Neurosci 21: 434-443. Medline

Clelland CD, Choi M, Romberg C, Clemenson GD Jr, Fragniere A, Tyers P, Jessberger S, Saksida LM, Barker RA, Gage FH, Bussey TJ (2009) A functional role for adult hippocampal neurogenesis in spatial pattern separation. Science 325:210-213. CrossRef Medline

Creer DJ, Romberg C, Saksida LM, van Praag H, Bussey TJ (2010) Running enhances spatial pattern separation in mice. Proc Natl Acad Sci U S A 107:2367-2372. CrossRef Medline
Cui Z, Wang H, Tan Y, Zaia KA, Zhang S, Tsien JZ (2004) Inducible and reversible NR1 knockout reveals crucial role of the NMDA receptor in preserving remote memories in the brain. Neuron 41:781-793. CrossRef Medline

Cundiff P, Liu L, Wang Y, Zou J, Pan YW, Abel G, Duan X, Ming GL, Englund C, Hevner R, Xia Z (2009) ERK5 MAP kinase regulates Neurogenin1 during cortical neurogenesis. PLoS One 4:e5204. CrossRef Medline

David DJ, Samuels BA, Rainer Q, Wang JW, Marsteller D, Mendez I, Drew M, Craig DA, Guiard BP, Guilloux JP, Artymyshyn RP, Gardier AM, Gerald C, Antonijevic IA, Leonardo ED, Hen R (2009) Neurogenesisdependent and -independent effects of fluoxetine in an animal model of anxiety/depression. Neuron 62:479-493. CrossRef Medline

Deng W, Saxe MD, Gallina IS, Gage FH (2009) Adult-born hippocampal dentate granule cells undergoing maturation modulate learning and memory in the brain. J Neurosci 29:13532-13542. CrossRef Medline

Deng W, Aimone JB, Gage FH (2010) New neurons and new memories: how does adult hippocampal neurogenesis affect learning and memory? Nat Rev Neurosci 11:339-350. CrossRef Medline

Eckel-Mahan KL, Phan T, Han S, Wang H, Chan GC, Scheiner ZS, Storm DR (2008) Circadian oscillation of MAPK activity and cAMP in the hippocampus: implications for memory persistence. Nat Neurosci 11:10741082. CrossRef Medline

English JM, Vanderbilt CA, Xu S, Marcus S, Cobb MH (1995) Isolation of MEK5 and differential expression of alternatively spliced forms. J Biol Chem 270:28897-28902. CrossRef Medline

George SH, Gertsenstein M, Vintersten K, Korets-Smith E, Murphy J, Stevens ME, Haigh JJ, Nagy A (2007) Developmental and adult phenotyping directly from mutant embryonic stem cells. Proc Natl Acad Sci U S A 104:4455-4460. CrossRef Medline

Haettig J, Stefanko DP, Multani ML, Figueroa DX, McQuown SC, Wood MA (2011) HDAC inhibition modulates hippocampus-dependent longterm memory for object location in a CBP-dependent manner. Learn Mem 18:71-79. CrossRef Medline

Hayashi M, Lee JD (2004) Role of the BMK1/ERK5 signaling pathway: lessons from knockout mice. J Mol Med 82:800-808. CrossRef Medline

Imayoshi I, Sakamoto M, Ohtsuka T, Takao K, Miyakawa T, Yamaguchi M, Mori K, Ikeda T, Itohara S, Kageyama R (2008) Roles of continuous neurogenesis in the structural and functional integrity of the adult forebrain. Nat Neurosci 11:1153-1161. CrossRef Medline

Kato Y, Kravchenko VV, Tapping RI, Han J, Ulevitch RJ, Lee JD (1997) BMK1/ERK5 regulates serum-induced early gene expression through transcription factor MEF2C. EMBO J 16:7054-7066. CrossRef Medline

Kempermann G, Kuhn HG, Gage FH (1997) More hippocampal neurons in adult mice living in an enriched environment. Nature 386:493-495. CrossRef Medline

Kuo CT, Mirzadeh Z, Soriano-Navarro M, Rasin M, Wang D, Shen J, Sestan N, Garcia-Verdugo J, Alvarez-Buylla A, Jan LY, Jan YN (2006) Postnatal deletion of Numb/Numblike reveals repair and remodeling capacity in the subventricular neurogenic niche. Cell 127:1253-1264. CrossRef Medline

Lagace DC, Whitman MC, Noonan MA, Ables JL, DeCarolis NA, Arguello AA, Donovan MH, Fischer SJ, Farnbauch LA, Beech RD, DiLeone RJ, Greer CA, Mandyam CD, Eisch AJ (2007) Dynamic contribution of nestin-expressing stem cells to adult neurogenesis. J Neurosci 27:1262312629. CrossRef Medline

Lee AK, Wilson MA (2002) Memory of sequential experience in the hippocampus during slow wave sleep. Neuron 36:1183-1194. CrossRef Medline

Li T, Pan YW, Wang W, Abel G, Zou J, Xu L, Storm DR, Xia Z (2013) Targeted deletion of the ERK5 MAP kinase impairs neuronal differentiation, migration, and survival during adult neurogenesis in the olfactory bulb. PloS One 8:e61948. CrossRef Medline

Liu L, Cavanaugh JE, Wang Y, Sakagami H, Mao Z, Xia Z (2003) ERK5 activation of MEF2-mediated gene expression plays a critical role in BDNF-promoted survival of developing but not mature cortical neurons. Proc Natl Acad Sci U S A 100:8532-8537. CrossRef Medline

Liu L, Cundiff P, Abel G, Wang Y, Faigle R, Sakagami H, Xu M, Xia Z (2006) Extracellular signal-regulated kinase (ERK) 5 is necessary and sufficient to specify cortical neuronal fate. Proc Natl Acad Sci U S A 103:9697-9702. CrossRef Medline

Luo J, Phan TX, Yang Y, Garelick MG, Storm DR (2013) Increases in cAMP, MAPK activity, and CREB phosphorylation during REM sleep: implica- 
tions for REM sleep and memory consolidation. J Neurosci 33:64606468. CrossRef Medline

Madisen L, Zwingman TA, Sunkin SM, Oh SW, Zariwala HA, Gu H, Ng LL, Palmiter RD, Hawrylycz MJ, Jones AR, Lein ES, Zeng H (2010) A robust and high-throughput Cre reporting and characterization system for the whole mouse brain. Nat Neurosci 13:133-140. CrossRef Medline

Marín-Burgin A, Mongiat LA, Pardi MB, Schinder AF (2012) Unique processing during a period of high excitation/inhibition balance in adultborn neurons. Science 335:1238-1242. CrossRef Medline

Ming GL, Song H (2011) Adult neurogenesis in the mammalian brain: significant answers and significant questions. Neuron 70:687-702. CrossRef Medline

Okamura N, Garau C, Duangdao DM, Clark SD, Jüngling K, Pape HC, Reinscheid RK (2011) Neuropeptide S enhances memory during the consolidation phase and interacts with noradrenergic systems in the brain. Neuropsychopharmacology 36:744-752. CrossRef Medline

Oliveira AM, Hawk JD, Abel T, Havekes R (2010) Post-training reversible inactivation of the hippocampus enhances novel object recognition memory. Learn Mem 17:155-160. CrossRef Medline

Pan YW, Storm DR, Xia Z (2012a) The maintenance of established remote contextual fear memory requires ERK5 MAP kinase and ongoing adult neurogenesis in the hippocampus. PLoS One 7:e50455. CrossRef Medline

Pan YW, Kuo CT, Storm DR, Xia Z (2012b) Inducible and targeted deletion of the ERK5 MAP kinase in adult neurogenic regions impairs adult neurogenesis in the olfactory bulb and several forms of olfactory behavior. PLoS One 7:e49622. CrossRef Medline

Pan YW, Chan GC, Kuo CT, Storm DR, Xia Z (2012c) Inhibition of adult neurogenesis by inducible and targeted deletion of ERK5 mitogenactivated protein kinase specifically in adult neurogenic regions impairs contextual fear extinction and remote fear memory. J Neurosci 32:64446455. CrossRef Medline

Pan YW, Zou J, Wang W, Sakagami H, Garelick MG, Abel G, Kuo CT, Storm DR, Xia Z (2012d) Inducible and conditional deletion of extracellular signal-regulated kinase 5 disrupts adult hippocampal neurogenesis. J Biol Chem 287:23306-23317. CrossRef Medline

Petrik D, Jiang Y, Birnbaum SG, Powell CM, Kim MS, Hsieh J, Eisch AJ (2012) Functional and mechanistic exploration of an adult neurogenesis-promoting small molecule. FASEB J 26:3148-3162. CrossRef Medline

Sahay A, Scobie KN, Hill AS, O’Carroll CM, Kheirbek MA, Burghardt NS,
Fenton AA, Dranovsky A, Hen R (2011) Increasing adult hippocampal neurogenesis is sufficient to improve pattern separation. Nature 472:466470. CrossRef Medline

Shimizu K, Phan T, Mansuy IM, Storm DR (2007) Proteolytic degradation of SCOP in the hippocampus contributes to activation of MAP kinase and memory. Cell 128:1219-1229. CrossRef Medline

Shors TJ, Miesegaes G, Beylin A, Zhao M, Rydel T, Gould E (2001) Neurogenesis in the adult is involved in the formation of trace memories. Nature 410:372-376. CrossRef Medline

Soriano P (1999) Generalized lacZ expression with the ROSA26 Cre reporter strain. Nat Genet 21:70-71. CrossRef Medline

Tronche F, Kellendonk C, Kretz O, Gass P, Anlag K, Orban PC, Bock R, Klein R, Schütz G (1999) Disruption of the glucocorticoid receptor gene in the nervous system results in reduced anxiety. Nat Genet 23:99-103. CrossRef Medline

van Praag H, Kempermann G, Gage FH (1999) Running increases cell proliferation and neurogenesis in the adult mouse dentate gyrus. Nat Neurosci 2:266-270. CrossRef Medline

Wang J, Gallagher D, DeVito LM, Cancino GI, Tsui D, He L, Keller GM, Frankland PW, Kaplan DR, Miller FD (2012) Metformin activates an atypical PKC-CBP pathway to promote neurogenesis and enhance spatial memory formation. Cell Stem Cell 11:23-35. CrossRef Medline

Wang W, Pan YW, Wietecha T, Zou J, Abel GM, Kuo CT, Xia Z (2013) Extracellular signal-regulated kinase 5 (ERK5) mediates prolactinstimulated adult neurogenesis in the subventricular zone and olfactory bulb. J Biol Chem 288:2623-2631. CrossRef Medline

Watson FL, Heerssen HM, Bhattacharyya A, Klesse L, Lin MZ, Segal RA (2001) Neurotrophins use the Erk5 pathway to mediate a retrograde survival response. Nat Neurosci 4:981-988. CrossRef Medline

Zhao C, Deng W, Gage FH (2008) Mechanisms and functional implications of adult neurogenesis. Cell 132:645-660. CrossRef Medline

Zhou G, Bao ZQ, Dixon JE (1995) Components of a new human protein kinase signal transduction pathway. J Biol Chem 270:12665-12669. CrossRef Medline

Zou J, Pan YW, Wang Z, Chang SY, Wang W, Wang X, Tournier C, Storm DR, Xia Z (2012) Targeted deletion of ERK5 MAP kinase in the developing nervous system impairs development of GABAergic interneurons in the main olfactory bulb and behavioral discrimination between structurally similar odorants. J Neurosci 32:4118-4132. CrossRef Medline 